ESAIM: M2AN

Vol. 40, $\mathrm{N}^{\circ} 1,2006$, pp. 63-97

DOI: $10.1051 / \mathrm{m} 2 \mathrm{an}: 2006008$
ESAIM: Mathematical Modelling and Numerical Analysis

www.edpsciences.org/m2an

\title{
ASYMPTOTIC ANALYSIS OF AN APPROXIMATE MODEL FOR TIME HARMONIC WAVES IN MEDIA WITH THIN SLOTS
}

\author{
PATRICK Joly ${ }^{1}$ AND SÉBAstien TORDEuX ${ }^{2}$
}

\begin{abstract}
In this article, we derive a complete mathematical analysis of a coupled 1D-2D model for $2 \mathrm{D}$ wave propagation in media including thin slots. Our error estimates are illustrated by numerical results.
\end{abstract}

Mathematics Subject Classification. 35J05, 74J05, 78A45, 78M30, 78M35.

Received: May 23, 2005.

\section{IntRoduction}

In practical applications concerning both electromagnetic or acoustic waves, many wave propagation problems involve the presence of structures whose at least one space dimension of characteristic length, denoted $\varepsilon$, is small with respect to the wave length $\lambda$ : we think for instance to highly oscillating coefficients, thin layers, thin screens, wires or thin slots, which is the topic we address in this work. For the numerical simulation of such wave propagation problems, it is natural to look for approximate or "effective" models that should permit to avoid to mesh the computational domain, even locally, at the scale of $\varepsilon$. In the first three cases, there exist well established mathematical techniques that permit to construct the effective models:

- the homogeneization theory $[6,34]$ for highly heterogeneous media;

- the equivalent boundary (or transmission) conditions for thin layers $[2,5,15,35]$;

- the boundary integral equation theory for thin screens $[1,7]$.

In the case of wires, there exist so-called wire-approximation models that have been developed in particular by the electric engineering community (see [23,36,37] and references therein) but the mathematical justification of such models appears as much less mature than in the three previous situations (let us mention however [10] or [33]). One meets a similar situation for the treatment of thin slots. Let us take the example of a thin slot in a $2 \mathrm{D}$ context. The geometry of the problem is represented by Figure 1. A first application is the microwave shielding of thin slots. From the physical point of view, the domain of validity is typically given by:

$$
\lambda / 1000<\varepsilon<\lambda / 10, \quad \varepsilon<L / 10 \text { and } \lambda / 10<L<10 \lambda,
$$

where $\lambda$ is the wave length, $\varepsilon$ is the width of the slot and $L$ the length of the slot. One can refer to [38] for examples. A second application in electromagnetism corresponds to the so-called flanged waveguide antenna

Keywords and phrases. Slit, slot, wave equation, Helmholtz equation, approximate model.

1 UMR POEMS, CNRS-INRIA, France. patrick.joly@inria.fr

2 Seminar für Angewandte Mathematik, ETHZ, Switzerland. tordeux@math.ethz.ch 


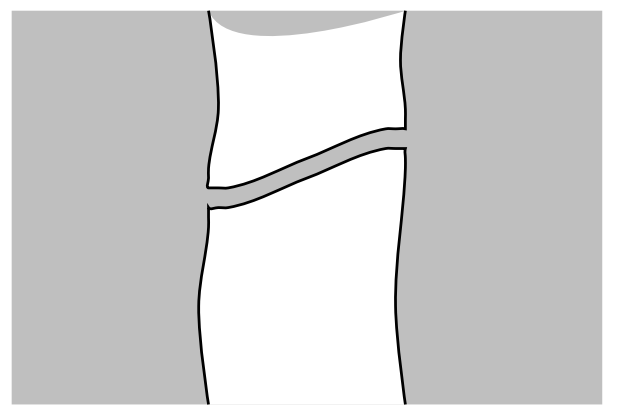

FiguRE 1. Geometry of the propagation domain.

which corresponds to a semi-infinite straight slot. This case, that has been studied in [26] in a non asymptotic context, will be analyzed in detail in this paper.

A intuitive idea is to try to derive an approximate "1D - 2D" model: a 1D model, posed on the curve that materializes the limit of the slot when $\varepsilon$ tends to 0 , for the propagation inside the slot and a $2 \mathrm{D}$ model for the rest of the computational domain. Such models have been designed in the engineering literature (see $[8,16,22,38]$ or [36] for a review) and are commonly used in various computational codes. However, the complete understanding and evaluation of such models suffers, to our opinion, from the following facts:

- These models are very often described at the discrete level, i.e. at the level of the numerical method that is used, and it is not always clear to identify the limit problem that one solves when the discretization parameters tend to 0 .

- At the continuous level, it is not difficult to design the appropriate propagation equations for the $1 \mathrm{D}$ and 2D models. What is less clear is the way (not unique - see Rem. 2.1) one couples the two models.

- Even when the continuous approximate model is identified, there is no mathematical result, to our knowledge, that permits to evaluate the error generated by this model.

Our objective is to fill partially the gap between the theory and the application. Mathematical problems of the same nature appear in mechanics, in particular in the theory of junctions (between a 3D solid and a thin plate for instance). However, the mathematical results developed in [9] or [27] are not of the same nature that the ones we shall look for in this article. Concerning wave propagation problems, one can cite some works on the asymptotic behaviour of eigenvalues [12] or resonances [4] in geometrical situations similar to the ones we shall consider here. The mathematical work that is the closest to ours corresponds to the Ph.D. thesis of Tatout (work which has not been published). In [39], from the theoretical point of view it obtains in particular some a priori estimates independent on $\varepsilon$ which are in the same spirit (although different) from the stability results we shall establish. From the numerical point of view, he derived a finite volume scheme with a special 1D treatment of the slot but did not study the quality of this model from the mathematical point of view.

To be complete, we must mention the analytical work by the "British school" of applied mathematics on this type of problem $[13,20,31,41]$. The aim they pursue a priori is not to derive approximate models for numerical approximation. They wish to describe as accurately as possible the behaviour of the solution with the help of analytical formulas or at least solutions of simple canonical problems. The technique that is used is the method of matched asymptotics expansions which consists in separating the domain of propagation in several parts in which one makes different ansatz on the solution. For instance, for the slot problem, it would consist in considering three zones: the inner slot, the exterior region and a neighborhood of the end points of the slot. Such techniques permit for instance to take correctly into account the singularities of the solution. Let us mention however that, here again, the developments are essentially formal and that some analysis is needed to justify them from a functional analytic point of view. We also think that this type of technique could be used to derive effective models for numerical computation and we are currently working in this direction. 
In this work, we shall consider the simplest possible propagation model, namely the 2D scalarwave equation, that can be used in acoustics of course, but also in electromagnetism if one separates the transverse electric or transverse magnetic polarizations:

$$
\frac{\partial^{2} U}{\partial t^{2}}-\Delta U=F
$$

where we have assumed, for simplicity, that the propagation velocity of waves is equal to 1 . We shall look at the time harmonic regime, i.e. consider a source term of the form:

$$
F(x, t)=f(x) \cdot \exp (-i \omega t) .
$$

where the pulsation $\omega>0$ is a data of the problem, related to the wavelength $\lambda$ by:

$$
\omega \lambda=2 \pi
$$

In (1.3), $f$ denotes a compactly supported function whose support does not intersect the slot. We then look for solutions of the same form:

$$
U(x, t)=u(x) \cdot \exp (-i \omega t),
$$

which leads to the Helmholtz equation for the complex value function $u$ :

$$
-\Delta u-\omega^{2} u=f .
$$

A typical source in application is a regularized $\delta$-function an approximation to a point source.

For the boundary condition, we shall consider the homogeneous Neumann condition:

$$
\frac{\partial u}{\partial n}=0 .
$$

In some sense, it is the "only" boundary condition that permits the propagation of waves inside the slot. This this due to the fact that the slot behaves as a waveguide whose first cut-off frequency is 0 .

This model is physically relevant. In acoustics, it corresponds to the so called sound hard boundary condition [11]. In 2D electromagnetism (or looks for solutions independent of the space variable $z$ ), the Maxwell system, satisfied by the electric field $\left(E_{x}, E_{y}, E_{z}\right)(x, y)$ and the electromagnetic field $\left(B_{x}, B_{y}, B_{z}\right)(x, y)$ with the perfectly conducting boundary condition - see [32] -, can be decoupled into two systems. One is relative to $\left(E_{x}, E_{y}, B_{z}\right)$ and the other one is relative to $\left(B_{x}, B_{y}, E_{z}\right)$. Each of these systems can be reduced to a scalar system satisfied by $E_{z}$ and $B_{z}$, which are transverse to the plane. They both satisfy a Helmholtz equation with the Dirichlet boundary condition for the transverse electric field $E_{z}$ and the Neumann boundary condition for the transverse magnetic field $B_{z}$. Hence, the field $B_{z}$ is solution of our model. One can refer to [3] for more details.

The outline of our article is as follows. In Section 2, we present the formal derivation of the approximate model and insist in particular on the variational nature of the coupling condition we choose. We also describe the model problem - namely the half-space problem with a semi-infinite straight slot - that will be the object of the mathematical analysis. In Section 3, we state our main results which concern first the asymptotic analysis of the exact solution when $\varepsilon$ tends to 0 (Sect. 3.1) and error estimates between the exact solution and the approximate one (Sect. 3.2). Section 4 is devoted to the proof of the results describing the asymptotic behaviour of the exact solution (Sect. 4.1) and of the approximate solution (Sect. 4.2). In Section 5, we prove the error estimates announced in Section 3.2. Finally in Section 6, we present numerical results that validate the theoretical ones.

\section{Derivation of the APproximate MOdel}

\subsection{The general case of a finite slot}

Let us first describe the geometry of the problem and introduce the notation. We assume that we are in 2D $\left(\mathbf{x}=(x, y)\right.$ will denote the current point of $\left.\mathbb{R}^{2}\right)$ and that the domain of propagation $\Omega_{\varepsilon}$ is the connected union 


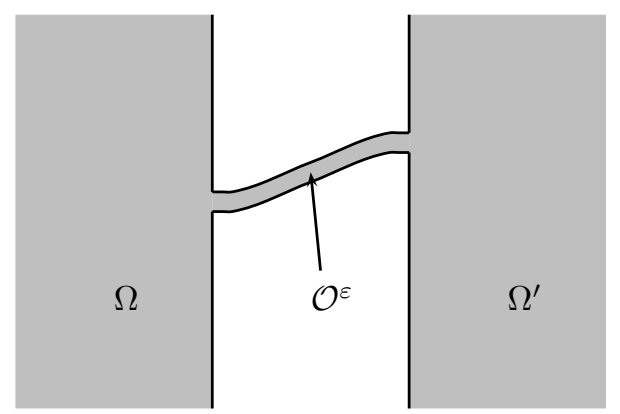

FIGURE 2. Geometry of the problem and notation.

of three domains:

$$
\bar{\Omega}_{\varepsilon}=\bar{\Omega} \cup \overline{\mathcal{O}}_{\varepsilon} \cup \bar{\Omega}^{\prime}
$$

where $\Omega$ and $\Omega^{\prime}$ are two parallel half-spaces distant from $L>0$ :

$$
\Omega=]-\infty, 0\left[\times \mathbb{R}, \quad \Omega^{\prime}=\right] L,+\infty[\times \mathbb{R}
$$

and where $\mathcal{O}_{\varepsilon}$ is a curved slot of width $\varepsilon$ that joins the two half-spaces, according to Figure 2 . To be more precise, we need to introduce some geometry. We start from a curve $\mathcal{C}$ of finite length $\widetilde{L} \geq L$, parameterized with its curvilinear abscissa $s$ :

$$
\mathcal{C}=\{\mathbf{x}=F(s), s \in] 0, \widetilde{L}[\},
$$

where the function $F=\left(F_{x}, F_{y}\right)^{t} \in C^{2}\left([0, \widetilde{L}] ; \mathbb{R}^{2}\right)$ verifies:

$$
\begin{cases}(i) & \forall s \in] 0, \widetilde{L}\left[, \quad\left|F^{\prime}(s)\right|=1,\right. \\ (i i) & F(0)=A \equiv(0,0)^{t}, \quad F(\widetilde{L})=B \equiv(L, h)^{t} \quad \text { for some } h \in \mathbb{R}, \\ (i i i) & F^{\prime}(0)=F^{\prime}(\widetilde{L})=(1,0), \\ (i v) \quad F \text { is injective from }] 0, \widetilde{L}[\text { in }] 0, L[\times \mathbb{R} .\end{cases}
$$

In (2.4), (ii) indicates that $\mathcal{C}$ connects $\Omega$ and $\Omega^{\prime}$ (with connecting points $A$ and $B$ ), (iii) indicates that $\mathcal{C}$ is normal to $\Omega$ and $\Omega^{\prime}$ and $(i v)$ means that $\mathcal{C}$ has no double points and does not penetrate $\Omega$ or $\Omega^{\prime}$. We introduce the tangent and normal vectors to $\mathcal{C}$ at point $F(s)$,

$$
t(s)=F^{\prime}(s)=\left(F_{x}^{\prime}(s), F_{y}^{\prime}(s)\right), \quad n(s)=\left(-F_{y}^{\prime}(s), F_{x}^{\prime}(s)\right),
$$

and the curvature $c(s)$ (with corresponding radius of curvature $R(s)=|c(s)|^{-1}$ ):

$$
c(s)=F_{x}^{\prime \prime}(s) F_{y}^{\prime}(s)-F_{x}^{\prime}(s) F_{y}^{\prime \prime}(s) .
$$

We denote $R_{*}$ the minimum radius of curvature:

$$
R_{*}=\min _{s \in] 0, \widetilde{L}[} R(s) \quad>0 .
$$




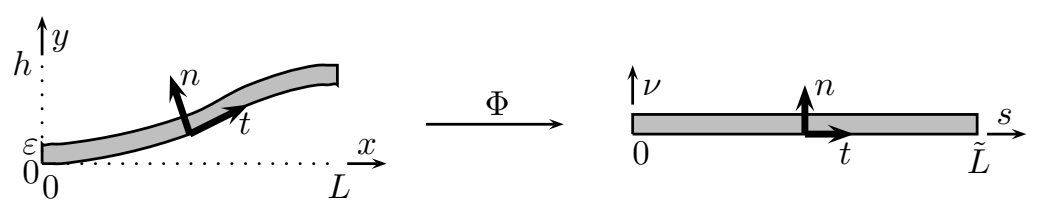

Figure 3. Parametrization of the curved slot.

For a given $\varepsilon>0$, we introduce the transformation:

$$
\begin{aligned}
\Phi:] 0, \widetilde{L}[\times] 0, \varepsilon[ & \longrightarrow \mathbb{R}^{2}, \\
(s, \nu) & \longmapsto F(s)+\nu n(s),
\end{aligned}
$$

whose Jacobian is given by $J_{\Phi}(s, \nu)=1-\nu c(s)$. Therefore, as soon as $\varepsilon<R_{*}$, $\Phi$ is injective and we can define the open $\operatorname{set} \mathcal{O}_{\varepsilon}$ as:

$$
\mathcal{O}_{\varepsilon}=\{x=\Phi(s, \nu), s \in] 0, \widetilde{L}[, \nu \in] 0, \varepsilon[\} .
$$

The pair $(s, \nu)$ constitutes a local system of coordinates in $\mathcal{O}_{\varepsilon}$ (see Fig. 3 ) and $\Phi$ is a bijection from $] 0, \widetilde{L}[\times] 0, \varepsilon[$ onto $\mathcal{O}_{\varepsilon}$. In particular, a function $u$ defined in $\mathcal{O}_{\varepsilon}$ can be identified to a function $u(s, \nu)$.

We assume, to simplify (this is not restrictive), that the propagation medium is a homogeneous medium with velocity $c=1$. We assume that a time harmonic wave of pulsation $\omega>0$ is emitted by a source term $f$ with compact support inside the half-space $\Omega$. Thus, the solution $u^{\varepsilon}$ inside $\Omega_{\varepsilon}$ obeys the following equations:

$$
\begin{cases}-\Delta u^{\varepsilon}-\omega^{2} u^{\varepsilon}=f, & \text { in } \Omega_{\varepsilon} \\ \frac{\partial u^{\varepsilon}}{\partial n}=0, & \text { on } \partial \Omega_{\varepsilon}\end{cases}
$$

as well as the outgoing radiation condition at infinity $\left(S_{R}\right.$ denotes the circle of radius $\left.R\right)$ :

$$
\lim _{R \rightarrow+\infty} \int_{S_{R} \cap \Omega_{\varepsilon}}\left|\frac{\partial u^{\varepsilon}}{\partial r}-i \omega u^{\varepsilon}\right|^{2} \mathrm{~d} \sigma=0, \quad(r=|\mathbf{x}|) .
$$

It is well known that problem $((2.10),(2.11))$ admits, for any $f \in L^{2}(\Omega)$ with compact support, a unique solution $u^{\varepsilon} \in H_{l o c}^{1}\left(\Omega_{\varepsilon}\right)$. Moreover, for both practical and theoretical reasons, the problem can be reduced to a bounded domain (see Fig. 4), by introducing two artificial (semi-)circular boundaries:

$$
\left\{\begin{array}{l}
\Gamma_{R}=\{\mathbf{x}=(x, y) \in \Omega /|\mathbf{x}-A|=R\}, \\
\Gamma_{R}^{\prime}=\left\{\mathbf{x}=(x, y) \in \Omega^{\prime} /|\mathbf{x}-B|=R\right\} .
\end{array}\right.
$$

We simply recall the results that are quite classical. Using separation of variables in polar coordinates $(r, \theta)$, it is possible to write exact boundary conditions on $\Gamma_{R}$ and $\Gamma_{R}^{\prime}$, as soon as $R$ is large enough so that the support of $f$ is included in $\Omega_{R}$ (see $(2.17)$ ):

$$
\begin{cases}\frac{\partial u^{\varepsilon}}{\partial r}+T_{R}\left(u^{\varepsilon}\right)=0, & \text { on } \Gamma_{R} \\ \frac{\partial u^{\varepsilon}}{\partial r}+T_{R}^{\prime}\left(u^{\varepsilon}\right)=0, & \text { on } \Gamma_{R}^{\prime}\end{cases}
$$




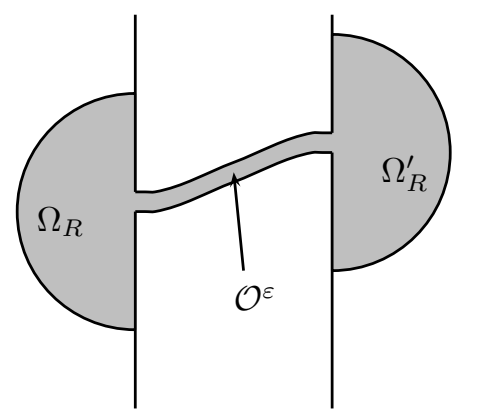

FigurE 4. The truncated domain.

where $T_{R} \in \mathcal{L}\left(H^{1 / 2}\left(\Gamma_{R}\right), H^{-1 / 2}\left(\Gamma_{R}\right)\right)$ and $T_{R}^{\prime} \in \mathcal{L}\left(H^{1 / 2}\left(\Gamma_{R}^{\prime}\right), H^{-1 / 2}\left(\Gamma_{R}^{\prime}\right)\right)$ are two non local (DtN) maps that are known explicitly [25]. For instance, for $T_{R}$ (it is the "same" for $T_{R}^{\prime}$ ), if the semi-circle $\Gamma_{R}$ is parameterized by $\theta \in] 0, \pi[$, one has:

$$
T_{R}: \phi(\theta)=\sum_{n=0}^{+\infty} \phi_{n} \psi_{n}(\theta) \quad \mapsto \quad T_{R} \phi(\theta)=\sum_{n=0}^{+\infty} \mu_{n}^{R}(\omega) \phi_{n} \psi_{n}(\theta)
$$

where we have set:

$$
\left\{\begin{array}{l}
\psi_{0}(\theta)=\sqrt{\frac{1}{R}}, \quad \psi_{n}(\theta)=\sqrt{\frac{2}{R}} \cos (n \theta), \quad \forall n \in \mathbb{N}^{*}, \\
\mu_{n}^{R}(\omega)=-\omega \frac{H_{n}^{(1)^{\prime}}(\omega R)}{H_{n}^{(1)}(\omega R)}, \quad \forall n \in \mathbb{N} .
\end{array}\right.
$$

In $(2.15), H_{n}^{(1)}$ is the classical first kind Hankel function [28]. We recall the the functions $\left\{\psi_{n}, n \geq 0\right\}$ form a basis of $H^{s}\left(\Gamma_{R}\right)$ for any $s<3 / 2$ [14], which is orthonormal in $L^{2}\left(\Gamma_{R}\right)$. Of course, the use of the radiation conditions at finite distance (2.13), permits us to reduce the problem to:

$$
\Omega_{\varepsilon}^{R}=\Omega_{R} \cup \mathcal{O}_{\varepsilon} \cup \Omega_{R}^{\prime}
$$

where

$$
\left\{\begin{array}{l}
\Omega_{R}=\{\mathbf{x}=(x, y) \in \Omega /|\mathbf{x}-A|<R\} \\
\Omega_{R}^{\prime}=\left\{\mathbf{x}=(x, y) \in \Omega^{\prime} /|\mathbf{x}-B|<R\right\}
\end{array}\right.
$$

The idea for the construction of the approximate model simply consists in assuming that, as a first approximation, the solution remains constant in any section $s=$ constant of the slot. In other words, one makes the approximation:

$$
u^{\varepsilon}(s, \nu) \sim U^{\varepsilon}(s)=\frac{1}{\varepsilon} \int_{0}^{\varepsilon} u^{\varepsilon}(s, \nu) \mathrm{d} \nu, \quad(\varepsilon \rightarrow 0) .
$$

To obtain an equation for $U^{\varepsilon}$, one integrates with respect to $\nu$ the Hemholtz equation, written in curvilinear coordinates:

$$
-\frac{\partial}{\partial s}\left([1+\nu c(s)]^{-1} \frac{\partial u^{\varepsilon}}{\partial s}\right)-\frac{\partial}{\partial \nu}\left([1+\nu c(s)] \frac{\partial u^{\varepsilon}}{\partial \nu}\right)-\omega^{2}[1+\nu c(s)] u^{\varepsilon}=0 .
$$

Taking into account the boundary conditions:

$$
\frac{\partial u^{\varepsilon}}{\partial \nu}(s, 0)=\frac{\partial u^{\varepsilon}}{\partial \nu}(s, \varepsilon)=0
$$


and using the approximation (2.18), we get the 1D equation:

$$
-\frac{\partial}{\partial s}\left(\frac{1}{\varepsilon c(s)} \log [1+\varepsilon c(s)] \frac{\partial U^{\varepsilon}}{\partial s}\right)-\omega^{2}\left[1+\frac{\varepsilon c(s)}{2}\right] U^{\varepsilon}=0 .
$$

Let us set

$$
\Sigma_{\varepsilon}=\{(0, y) / 0<y<\varepsilon\} \subset \partial \Omega
$$

and

$$
\Sigma_{\varepsilon}^{\prime}=\{(L, y) / h<y<h+\varepsilon\} \subset \partial \Omega^{\prime} .
$$

We propose then to solve the following set of equations (defined in domains independent of $\varepsilon$ ):

$$
\left\{\begin{array}{l}
\text { Find } \left.\widetilde{u}^{\varepsilon}: \Omega \cup \Omega^{\prime} \rightarrow \mathbb{C} \text { and } U^{\varepsilon}: I=\right] 0, \widetilde{L}[\rightarrow \mathbb{C} \text { such that: } \\
-\Delta \widetilde{u}^{\varepsilon}-\omega^{2} \widetilde{u}^{\varepsilon}=f \text { in } \Omega \cup \Omega^{\prime}, \quad \frac{\partial \widetilde{u}^{\varepsilon}}{\partial n}=0 \text { on } \partial \Omega \backslash \Sigma_{\varepsilon} \cup \partial \Omega^{\prime} \backslash \Sigma_{\varepsilon}^{\prime}, \\
\widetilde{u}^{\varepsilon} \text { satisfies the radiation condition }(2.11) \text { at infinity, } \\
-\frac{\partial}{\partial s}\left(\frac{1}{\varepsilon c(s)} \log [1+\varepsilon c(s)] \frac{\partial U^{\varepsilon}}{\partial s}\right)-\omega^{2}\left(1+\frac{\varepsilon c(s)}{2}\right) U^{\varepsilon}=0, \quad s \in I,
\end{array}\right.
$$

that must be completed by some approximate "1D-2D transmission conditions" at points $A$ and $B$ that must be consistent with the continuity of $u^{\varepsilon}$ (Dirichlet) and $\partial u^{\varepsilon} / \partial x$ (Neumann) across $\Sigma_{\varepsilon}$ and $\Sigma_{\varepsilon}^{\prime}$. The (double) mathematical difficulty is that:

- $\Sigma_{\varepsilon}$ and $\Sigma_{\varepsilon}^{\prime}$ have no sense for $U^{\varepsilon}$ : only the points $A(s=0)$ and $B(s=\widetilde{L})$ make sense.

- Conversely, one can not speak of the value of $\widetilde{u}^{\varepsilon}$ or $\partial \widetilde{u}^{\varepsilon} / \partial x$ at points $A$ and $B$.

The solution, rather natural, that we propose consists in imposing the Dirichlet and Neumann transmission condition in a mean sense. For the Dirichlet condition, we simply write that:

$$
U^{\varepsilon}(0)=\frac{1}{\varepsilon} \int_{\Sigma_{\varepsilon}} \widetilde{u}^{\varepsilon} \mathrm{d} \sigma, \quad U^{\varepsilon}(\widetilde{L})=\frac{1}{\varepsilon} \int_{\Sigma_{\varepsilon}^{\prime}} \widetilde{u}^{\varepsilon} \mathrm{d} \sigma
$$

while for the Neumann condition we write that $\partial \widetilde{u}^{\varepsilon} / \partial x$ is constant along $\Sigma_{\varepsilon}$ and $\Sigma_{\varepsilon}^{\prime}$ :

$$
\frac{\partial \widetilde{u}^{\varepsilon}}{\partial n}=\frac{\mathrm{d} U^{\varepsilon}}{\mathrm{d} s}(0) \text { on } \Sigma_{\varepsilon}, \quad \frac{\partial \widetilde{u}^{\varepsilon}}{\partial n}=-\frac{\mathrm{d} U^{\varepsilon}}{\mathrm{d} s}(\widetilde{L}) \text { on } \Sigma_{\varepsilon}^{\prime} \text {. }
$$

The boundary value problem $((2.24),(2.25),(2.26))$, when reduced to a bounded domain thanks to conditions (2.13) admits a variational formulation in the Hilbert space:

$$
W_{\varepsilon}^{R}=\left\{\left(v^{\varepsilon}, V^{\varepsilon}\right) \in H^{1}\left(\Omega_{R} \cup \Omega_{R}^{\prime}\right) \times H^{1}(I) / V^{\varepsilon}(0)=\frac{1}{\varepsilon} \int_{\Sigma_{\varepsilon}} v^{\varepsilon} \mathrm{d} \sigma, V^{\varepsilon}(\tilde{L})=\frac{1}{\varepsilon} \int_{\Sigma_{\varepsilon}^{\prime}} v^{\varepsilon} \mathrm{d} \sigma\right\} .
$$

More precisely, it is equivalent to:

$$
\left\{\begin{array}{l}
\text { Find }\left(\widetilde{u}^{\varepsilon}, U^{\varepsilon}\right) \in W_{\varepsilon}^{R} \quad \text { such that, } \forall\left(v^{\varepsilon}, V^{\varepsilon}\right) \in W_{\varepsilon}^{R}: \\
a_{R}\left(\widetilde{u}^{\varepsilon}, v^{\varepsilon}\right)+b_{R}\left(\widetilde{u}^{\varepsilon}, v^{\varepsilon}\right)+b_{R}^{\prime}\left(\widetilde{u}^{\varepsilon}, v^{\varepsilon}\right)+\varepsilon a_{I}\left(U^{\varepsilon}, V^{\varepsilon}\right)=\int_{\Omega} f \bar{v}^{\varepsilon} \mathrm{d} \mathbf{x},
\end{array}\right.
$$


where we have set:

$$
\left\{\begin{array}{l}
a_{R}(u, v)=\int_{\Omega_{R} \cup \Omega_{R}^{\prime}}\left(\nabla u \cdot \nabla \bar{v}-\omega^{2} u \bar{v}\right) \mathrm{d} \mathbf{x} \\
a_{I}(U, V)=\int_{I}\left(\frac{\log [1+\varepsilon c(s)]}{\varepsilon c(s)} \frac{\mathrm{d} U}{\mathrm{~d} x} \frac{\mathrm{d} \bar{V}}{\mathrm{~d} x}-\omega^{2}\left(1+\frac{\varepsilon c(s)}{2}\right) U \bar{V}\right) \mathrm{d} x,
\end{array}\right.
$$

and where the bilinear form $b_{R}$ is given by (we omit the definition of $b_{R}^{\prime}$ which is similar):

$$
b_{R}(u, v)=\sum_{n=0}^{+\infty} \mu_{n}^{R}(\omega) u_{n}^{R} \bar{v}_{n}^{R}, \quad u_{n}^{R}=\int_{\Gamma_{R}} u \psi_{n} \mathrm{~d} \sigma, \quad v_{n}^{R}=\int_{\Gamma_{R}} v \psi_{n} \mathrm{~d} \sigma .
$$

We shall use later the following fundamental properties of the bilinear form $b_{R}$ (or $b_{R}^{\prime}$ ) which directly follow from the fact that all the numbers $\mu_{n}^{R}(\omega)$ have strictly positive real parts and strictly negative imaginary parts (This comes from the Wronskian of $J_{n}$ and $Y_{n}$ [28] and from the Nicholson formula [42]):

$$
\forall u \in H^{1}\left(\Omega_{R}\right), \quad \mathcal{R} e b_{R}(u, u) \geq 0, \quad \mathcal{I} m b_{R}(u, u) \leq 0 .
$$

Thanks to (2.30) is a simple exercise on Fredholm's alternative to show that (2.27) has a unique solution. Moreover, this problem can be treated numerically via the finite element method with computational meshes "ignoring" the space scale $\varepsilon$. Indeed, the geometry of the domain $\Omega \cup[0 ; \widetilde{L}]$ does not depend on $\varepsilon$. One can refer to [40] for more details.

Remark 2.1. As we said in the introduction, there are other ways than $((2.25),(2.26))$ to couple $\widetilde{u}^{\varepsilon}$ and $U^{\varepsilon}$. For instance, we can invert the roles of the Dirichlet and Neumann data and write:

$$
\begin{gathered}
\widetilde{u}^{\varepsilon}=U^{\varepsilon}(0) \text { on } \Sigma_{\varepsilon}, \quad \widetilde{u}^{\varepsilon}=U^{\varepsilon}(\widetilde{L}) \text { on } \Sigma_{\varepsilon}^{\prime}, \\
\frac{\mathrm{d} U^{\varepsilon}}{\mathrm{d} x}(0)=\frac{1}{\varepsilon} \int_{\Sigma_{\varepsilon}} \frac{\partial \widetilde{u}^{\varepsilon}}{\partial n} \mathrm{~d} \sigma, \quad \frac{\mathrm{d} U^{\varepsilon}}{\mathrm{d} x}(\widetilde{L})=-\frac{1}{\varepsilon} \int_{\Sigma_{\varepsilon}^{\prime}} \frac{\partial \widetilde{u}^{\varepsilon}}{\partial n} \mathrm{~d} \sigma .
\end{gathered}
$$

One easily checks that the (localized) variational formulation of $((2.24),(2.31),(2.32))$ is still given by (2.27) except that the space $W_{\varepsilon}^{R}$ has changed:

$$
W_{\varepsilon}^{R}=\left\{\left(v^{\varepsilon}, V^{\varepsilon}\right) \in H^{1}\left(\Omega_{R} \cup \Omega_{R}^{\prime}\right) \times H^{1}(I) / v^{\varepsilon}=V^{\varepsilon}(0) \text { on } \Sigma_{\varepsilon}, v^{\varepsilon}=V^{\varepsilon}(\tilde{L}) \text { on } \Sigma_{\varepsilon}^{\prime}\right\} .
$$

Of course, linear combinations between $((2.25),(2.26))$ and $((2.31),(2.32))$ provide new transmission conditions.

Remark 2.2. For the case of a straight slot, it is possible (and this is what we will do) to write a variational formulation only on the unknown $\widetilde{u}^{\varepsilon}$. The term $a_{I}$ is replaced by a Dirichlet to Neumann map associated to the slot problem.

\subsection{A model problem for the analysis; the semi-infinite problem}

For the mathematical analysis, we shall restrict the study to a simplified academic problem: the semi-infinite straight slot (see Fig. 5). With the notation of the previous section, this corresponds to:

$$
\widetilde{L}=+\infty, \quad s=x \text { and } F(s)=(s, 0)^{t}, \quad \Omega^{\prime}=\emptyset
$$




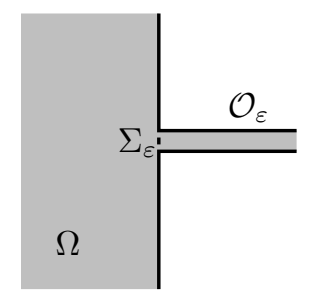

Figure 5. The semi infinite straight slot.

This problem has the advantage to present the main difficulty we wish to address in this paper, namely the "1D-2D" junction at point A. The equations that govern the exact solution are still given by $((2.10),(2.11))$. However, since this time the slot

$$
\left.\mathcal{O}_{\varepsilon}=\mathbb{R}^{+} \times\right] 0, \varepsilon\left[\quad\left(\Sigma_{\varepsilon}=\{0\} \times\right] 0 ; \varepsilon[)\right.
$$

is infinite in the $x$-direction, the outgoing radiation condition corresponds to selecting a certain category of solution inside the slot. To be more explicit, one has to exploit the separation of variables inside $\mathcal{O}_{\varepsilon}$. This naturally leads to introduce the sequence of functions (these are the eigenfunctions of the $1 \mathrm{D}$ operator $-d^{2} / d y^{2}$ in the segment $] 0, \varepsilon$ [ with homogeneous Neumann boundary conditions at $y=0$ and $y=\varepsilon$ ):

$$
w_{0}^{\varepsilon}(y)=\sqrt{\frac{1}{\varepsilon}} \quad \text { and } \quad w_{n}^{\varepsilon}(y)=\sqrt{\frac{2}{\varepsilon}} \cos \left(\frac{n \pi y}{\varepsilon}\right), \quad n=1,2,3, \ldots
$$

Let us assume that

$$
\varepsilon \omega<\pi
$$

which is automatically true for $\varepsilon$ small enough. As any solution of the homogeneous Helmholtz equation inside $\mathcal{O}_{\varepsilon}, u^{\varepsilon}$ is necessarily of the form:

$$
\left\{\begin{aligned}
u^{\varepsilon}(x, y)= & \left(a_{0}^{\varepsilon} \exp (i \omega x)+b_{0}^{\varepsilon} \exp (-i \omega x)\right) w_{0}^{\varepsilon}(y) \\
& +\sum_{n=1}^{+\infty}\left(a_{n}^{\varepsilon} \mathrm{e}^{-\xi_{n}^{\varepsilon}(\omega) x}+b_{n}^{\varepsilon} \mathrm{e}^{+\xi_{n}^{\varepsilon}(\omega) x}\right) w_{n}^{\varepsilon}(y),
\end{aligned}\right.
$$

where we have set:

$$
\xi_{n}^{\varepsilon}(\omega)=\sqrt{\frac{n^{2} \pi^{2}}{\varepsilon^{2}}-\omega^{2}}, \quad n \geq 1, \quad\left(\xi_{n}^{\varepsilon}(\omega)>0 \quad \text { thanks to }(2.36)\right) .
$$

Saying that $u^{\varepsilon}$ is an outgoing solution means that the coefficients $b_{n}^{\varepsilon}$ are necessarily 0 :

$$
u^{\varepsilon} \text { is outgoing } \Longrightarrow u^{\varepsilon}(x, y)=a_{0}^{\varepsilon} \exp (i \omega x) w_{0}^{\varepsilon}(y)+\sum_{n=1}^{+\infty} a_{n}^{\varepsilon} \mathrm{e}^{-\xi_{n}^{\varepsilon}(\omega) x} w_{n}^{\varepsilon}(y) \text {. }
$$

In other words, $u^{\varepsilon}$ appears as the superposition of:

- a single propagative mode: $a_{0}^{\varepsilon} \exp (i \omega x) w_{0}^{\varepsilon}(y)$;

- an infinity of evanescent (in the direction $x>0$ ) modes: $a_{n}^{\varepsilon} \mathrm{e}^{-\xi_{n}^{\varepsilon}(\omega) x} w_{n}^{\varepsilon}(y)$.

Remark 2.3. In the half space $\Omega$, we say that a solution of the Helmholtz equations is outgoing if:

$$
\lim _{R \rightarrow+\infty} \int_{\Gamma_{R}}\left|\frac{\partial u}{\partial r}-i \omega u\right|^{2} \mathrm{~d} \sigma=0 .
$$


For $R$ large enough so that the support of $f$ is included in $\Omega_{R}$ (see (2.17)), this is equivalent to:

$$
\frac{\partial u}{\partial n}+T_{R} u=0, \quad \text { on } \Gamma_{R} . \quad \text { (see (2.14) and (2.15)) }
$$

According to the previous section, the approximate problem that we shall consider can be written as (see (2.22) for $\left.\Sigma_{\varepsilon}\right)$ :

$$
\left\{\begin{array}{l}
\text { Find } \widetilde{u}^{\varepsilon}: \Omega \rightarrow \mathbb{C} \text { and } U^{\varepsilon}: \mathbb{R}^{+} \rightarrow \mathbb{C} \text { such that: } \\
-\Delta \widetilde{u}^{\varepsilon}-\omega^{2} \widetilde{u}^{\varepsilon}=f \text { in } \Omega, \quad \frac{\partial \widetilde{u}^{\varepsilon}}{\partial n}=0 \text { on } \partial \Omega \backslash \Sigma_{\varepsilon}, \\
-\frac{d^{2} U^{\varepsilon}}{\mathrm{d} x^{2}}-\omega^{2} U^{\varepsilon}=0, \text { on } \mathbb{R}^{+}, \\
U^{\varepsilon}(0)=\frac{1}{\varepsilon} \int_{\Sigma_{\varepsilon}} u^{\varepsilon} \mathrm{d} \sigma, \quad \frac{\partial \widetilde{u}^{\varepsilon}}{\partial n}=\frac{\mathrm{d} U^{\varepsilon}}{\mathrm{d} x}(0) \text { on } \Sigma_{\varepsilon},
\end{array}\right.
$$

completed by the fact that $\left(\widetilde{u}^{\varepsilon}, U^{\varepsilon}\right)$ must be "outgoing at infinity". For $\widetilde{u}^{\varepsilon}$, this is nothing but condition (2.11) (simply replace $\Omega_{\varepsilon}$ by $\Omega$ ). For $U^{\varepsilon}$, it is even simpler. Saying that $U^{\varepsilon}$ is outgoing simply amounts to saying that it is of the form:

$$
U^{\varepsilon}(x)=U^{\varepsilon}(0) \exp (i \omega x), \quad \forall x \in \mathbb{R}^{+} .
$$

To some extent, it also means that we have retained only the propagative mode in the series (2.39). Of course to define the approximate solution $\widetilde{u}^{\varepsilon}$ everywhere in $\Omega_{\varepsilon}$, we shall extend $\widetilde{u}^{\varepsilon}$ in $\mathcal{O}_{\varepsilon}$ by:

$$
\widetilde{u}^{\varepsilon}(x, y)=U^{\varepsilon}(x), \quad \text { in } \mathcal{O}_{\varepsilon} .
$$

In the remaining of the paper, our goal will be to estimate the difference $u^{\varepsilon}-\widetilde{u}^{\varepsilon}$ in $\Omega$ and $\mathcal{O}_{\varepsilon}$.

In this view, we shall introduce the problem satisfied by the limit $u^{0}$ of $u^{\varepsilon}$ in $\Omega$. As it is intuitive (and will be demonstrated in Sect. 3), this problem is the pure half-space problem in the absence of the slot:

$$
\begin{cases}-\Delta u^{0}-\omega^{2} u^{0}=f, & \text { in } \Omega, \\ \frac{\partial u^{0}}{\partial n}=0, & \text { on } \partial \Omega \\ u^{0} \text { is outgoing at infinity } & \text { (see Rem. 2.3). }\end{cases}
$$

This problem ignores the slot and consists in saying that no energy is transmitted inside $\mathcal{O}_{\varepsilon}$, which is not the case of the approximate (2.42). A relevant analysis must show that (2.42) provides a better approximation to the exact solution than (2.45). This implies to:

- compare $u^{\varepsilon}-\widetilde{u}^{\varepsilon}$ and $u^{\varepsilon}-u^{0}$ in $\Omega$;

- compare $u^{\varepsilon}-\widetilde{u}^{\varepsilon}$ and $u^{\varepsilon}$ in $\mathcal{O}_{\varepsilon}$.

This is the object of the rest of the paper.

\section{MAin RESUlts}

The object of Section 3.1 is to estimate the difference $u^{\varepsilon}-u^{0}$ in various subdomains of the half-space $\Omega$. It will be useful to differentiate the domains $\Omega_{R}$, whose closure contains the origin, from the domains $\Omega_{R, R^{\prime}}$ defined for $0<R^{\prime}<R$ by:

$$
\Omega_{R, R^{\prime}}=\left\{\mathbf{x}=(x, y) \in \Omega / R^{\prime}<|x|<R\right\} .
$$


One intuitive reason is linked to the fact that the function $u_{0}$ is smooth at the neighborhood of 0 while, for each $\varepsilon>0$, the exact solution has the maximal regularity (see [18] for instance):

$$
u^{\varepsilon} \in H^{\frac{5}{3}-\eta}\left(\Omega_{R}\right), \quad \text { for any } \eta>0 .
$$

This is due to the singularity in $r^{\frac{2}{3}}$ due to the presence of the two re-entrant corners of aperture $3 \pi / 2$. This makes us expect a different behaviour of $u^{\varepsilon}-u^{0}$ in $\Omega_{R}$ and $\Omega_{R, R^{\prime}}$.

In Section 3.2, we shall estimate the error $u^{\varepsilon}-\widetilde{u}^{\varepsilon}$ in the same domains $\Omega_{R}$ and $\Omega_{R, R^{\prime}}$. Note that $\widetilde{u}^{\varepsilon}$ is also singular in $\Omega$ but that its singularity differs from the one of $u^{\varepsilon}$. Notice that the restriction of $\widetilde{u}^{\varepsilon}$ is solution of a non homogeneous Neumann problem for the Helmholtz operator, the boundary data being equal - up to a multiplicative constant - to a characteristic function, which lives in $H^{\frac{1}{2}-\eta}(\Gamma)$. Hence once has:

$$
\widetilde{u}^{\varepsilon} \in H^{2-\eta}\left(\Omega_{R}\right), \quad \text { for any } \eta>0 .
$$

We shall also look at the error in bounded subdomains of the semi-infinite slot $\mathcal{O}_{\varepsilon}$. For any $0<L<L^{\prime}$, we set:

$$
\left\{\begin{array}{l}
\mathcal{O}_{\varepsilon}^{L}=\left\{\mathbf{x}=(x, y) \in \mathcal{O}_{\varepsilon} / x<L\right\}, \\
\mathcal{O}_{\varepsilon}^{L, L^{\prime}}=\left\{\mathbf{x}=(x, y) \in \mathcal{O}_{\varepsilon} / L^{\prime}<x<L\right\} .
\end{array}\right.
$$

Once again, we will see that we get different estimates in $\mathcal{O}_{\varepsilon}^{L}$ and $\mathcal{O}_{\varepsilon}^{L, L^{\prime}}$.

\subsection{Convergence to the limit problem}

Our first result says how fast $u^{\varepsilon}$, the exact solution approaches $u^{0}$, the limit solution.

Theorem 3.1. The function $u^{\varepsilon}$ converges to $u^{0}$ in $H^{1}\left(\Omega_{R}\right)$ when $\varepsilon$ tends to 0 and one has the error estimates, $C(R)$ denoting a positive constant:

$$
\begin{gathered}
\left\|u^{\varepsilon}-u^{0}\right\|_{H^{1}\left(\Omega_{R}\right)} \leq C(R) \varepsilon \sqrt{|\log \varepsilon|}\|f\|_{L^{2}(\Omega)} . \\
\left\|u^{\varepsilon}-u^{0}\right\|_{L^{2}\left(\Omega_{R}\right)} \leq C(R) \varepsilon\|f\|_{L^{2}(\Omega)} .
\end{gathered}
$$

Moreover, for any $0<R^{\prime}<R$, one has the improved error estimate:

$$
\left\|u^{\varepsilon}-u^{0}\right\|_{H^{1}\left(\Omega_{R, R^{\prime}}\right)} \leq C\left(R, R^{\prime}\right) \varepsilon\|f\|_{L^{2}(\Omega)},
$$

where $C\left(R, R^{\prime}\right)$ is, once again, a positive constant.

Remark 3.1. One can remark that:

- One gains only the factor $\sqrt{|\log \varepsilon|}$ by passing from the $H^{1}$ norm to the $L^{2}$ norm in $\Omega_{R}$ (see also Th. 3.2).

- One does not gain anything in the domains $\Omega_{R, R^{\prime}}$ as emphasized by Theorem 3.2.

Our next result aims at showing the optimality of the estimates of Theorem 3.1.

Theorem 3.2. For any $R>0$, there exist two strictly positive constants $C_{1}(R)$ and $C_{2}(R)$ such that:

$$
\begin{gathered}
\left\|u^{\varepsilon}-u^{0}\right\|_{H^{1}\left(\Omega_{R}\right)} \geqslant C_{1}(R) \varepsilon \sqrt{|\log (\varepsilon)|}\left|u^{0}(0,0)\right|-C_{2}(R) \varepsilon^{2}|\log (\varepsilon)|^{3 / 2}\|f\|_{L^{2}(\Omega)}, \\
\left\|u^{\varepsilon}-u^{0}\right\|_{L^{2}\left(\Omega_{R}\right)} \geqslant C_{1}(R) \varepsilon\left|u^{0}(0,0)\right|-C_{2}(R) \varepsilon^{2}|\log (\varepsilon)|\|f\|_{L^{2}(\Omega)} .
\end{gathered}
$$

Moreover, for any $0<R^{\prime}<R$, there exist two strictly positive constants $C_{1}\left(R, R^{\prime}\right)$ and $C_{2}\left(R, R^{\prime}\right)$ such that:

$$
\left\|u^{\varepsilon}-u^{0}\right\|_{L^{2}\left(\Omega_{R, R^{\prime}}\right)} \geqslant C_{1}\left(R, R^{\prime}\right) \varepsilon\left|u^{0}(0,0)\right|-C_{2}\left(R, R^{\prime}\right) \varepsilon^{2}|\log (\varepsilon)|\|f\|_{L^{2}(\Omega)} .
$$


Remark 3.2. The inequalities (3.8) to (3.10) prove that the estimates of Theorem 3.1 are optimal as soon as $u^{0}(0,0) \neq 0$, which is generically true. This is not the case, for instance, if $f$ is odd in $y$. However, we think that, in such a case, the estimates over $u^{\varepsilon}-u^{0}$ can be improved.

Remark 3.3. The initial problems are formulated in an unbounded domain $\Omega$, but the error estimates are stated in bounded domains. This is due to the fact that the solutions of the Helmholtz equation are not in $H^{1}(\Omega)$ or $L^{2}(\Omega)$.

\subsection{Error estimates for the approximate model}

We first give estimates in subdomains of the half-space $\Omega$.

Theorem 3.3. For any $R>0$, there exists a positive constant $C(R)$ such that:

$$
\left\|\widetilde{u}^{\varepsilon}-u^{\varepsilon}\right\|_{H^{1}\left(\Omega_{R}\right)} \leq C(R) \varepsilon\|f\|_{L^{2}(\Omega)} .
$$

For any $\delta>0$, there exists a positive constant $C(R, \delta)$ such that:

$$
\left\|\widetilde{u}^{\varepsilon}-u^{\varepsilon}\right\|_{L^{2}\left(\Omega_{R}\right)} \leq C(R, \delta) \varepsilon^{2-\delta}\|f\|_{L^{2}(\Omega)} .
$$

For any $0<R^{\prime}<R$, there exists a positive constant $C\left(R, R^{\prime}\right)$ such that:

$$
\left\|\widetilde{u}^{\varepsilon}-u^{\varepsilon}\right\|_{H^{1}\left(\Omega_{R, R^{\prime}}\right)} \leq C\left(R, R^{\prime}\right) \varepsilon^{2}\|f\|_{L^{2}(\Omega)} .
$$

Remark 3.4. Let us notice that:

- Contrary to what happens in $\Omega_{R}$, in the domains $\Omega_{R, R^{\prime}}$ one does not gain anything by passing from the $H^{1}$ norm to the $L^{2}$ norm: the estimate remains $\mathrm{O}\left(\varepsilon^{2}\right)$. Our conjecture is that such a result is generically optimal as in Theorem 3.2:

$$
\text { Conjecture: } \quad\left\|u^{\varepsilon}-\widetilde{u}^{\varepsilon}\right\|_{L^{2}\left(\Omega_{R, R^{\prime}}\right)} \geqslant C \varepsilon^{2} .
$$

We think this is also true for the estimates (3.11) and (3.13):

$$
\text { Conjecture: } \quad\left\{\begin{array}{l}
\left\|u^{\varepsilon}-\widetilde{u}^{\varepsilon}\right\|_{H^{1}\left(\Omega_{R}\right)} \geqslant C \varepsilon, \\
\left\|u^{\varepsilon}-\widetilde{u}^{\varepsilon}\right\|_{H^{1}\left(\Omega_{R, R^{\prime}}\right)} \geqslant C \varepsilon^{2},
\end{array}\right.
$$

and that estimate (3.12) is quasi-optimal:

$$
\text { Conjecture: } \quad\left\|u^{\varepsilon}-\widetilde{u}^{\varepsilon}\right\|_{L^{2}\left(\Omega_{R}\right)} \geqslant C \varepsilon^{2} .
$$

This will be confirmed by numerical results.

- It is easy to prove that the estimate (3.13) remains valid in any $H^{s}$-norm, $s>0$ :

$$
\left\|u^{\varepsilon}-\widetilde{u}^{\varepsilon}\right\|_{H^{s}\left(\Omega_{R, R^{\prime}}\right)} \leqslant C\left(s, R, R^{\prime}\right) \varepsilon^{2}\|f\|_{L^{2}(\Omega)}
$$

When one looks to higher order Sobolev norms in $\Omega_{R}$ (which is only possible for $s<5 / 3$ which corresponds to the maximal regularity of the exact solution $u^{\varepsilon}$ ), one can expect to lose powers of $\varepsilon$ :

$$
\text { Conjecture: }\left\{\begin{array}{l}
\left\|u^{\varepsilon}-\widetilde{u}^{\varepsilon}\right\|_{H^{s}\left(\Omega_{R, R^{\prime}}\right)} \leqslant C(s) \varepsilon^{g(s)}\|f\|_{L^{2}(\Omega)}, \forall s<5 / 3, \\
\text { with } C(s) \rightarrow+\infty \text { and } / \text { or } f(s) \rightarrow 0 \text { when } s \rightarrow \frac{5}{3} .
\end{array}\right.
$$


TABLE 1. Error estimates in the half-space.

\begin{tabular}{|c|c|c|c|c|}
\hline$V$ & $=$ & $H^{1}\left(\Omega_{R}\right)$ & $L^{2}\left(\Omega_{R}\right)$ & $H^{1}\left(\Omega_{R, R^{\prime}}\right)$ \\
\hline$\left\|u^{\varepsilon}-\widetilde{u}^{\varepsilon}\right\|_{V}$ & $\leqslant$ & $C(R) \varepsilon$ & $C(R, \delta) \varepsilon^{2-\delta}, \forall \delta>0$ & $C\left(R, R^{\prime}\right) \varepsilon^{2}$ \\
\hline$\frac{\left\|u^{\varepsilon}-\widetilde{u}^{\varepsilon}\right\|_{V}}{\left\|u^{\varepsilon}-u^{0}\right\|_{V}}$ & $\leqslant$ & $C(R)|\log (\varepsilon)|^{-1 / 2}$ & $C(R, \delta) \varepsilon^{1-\delta}, \forall \delta>0$ & $C\left(R, R^{\prime}\right) \varepsilon$ \\
\hline
\end{tabular}

We try to summarize the results of Theorems 3.1 and 3.2 in the following table. In particular, in the last line of the table, we study - in the case where $u_{0}(0,0) \neq 0$ - the gain in the order of convergence obtained by replacing $u^{0}$ by $\widetilde{u}^{\varepsilon}$. When one looks in domains containing the origin (the domains $\Omega_{R}$ ), this gain is only $O\left(|\log \varepsilon|^{-\frac{1}{2}}\right.$ ) in the $H^{1}$-norm and "almost" $O(\varepsilon)$ in the $L^{2}$-norm. This clearly traduces the fact that the singularity and the boundary layer of the exact solution is not well taken into account by the approximate one. In the domains $\Omega_{R, R^{\prime}}$, which do not contain the origin, this gain is $O(\varepsilon)$ in $H^{1}$-norm (in fact in any $H^{s}$-norm, $s \geq 0$ ).

Next we look at the errors in subdomains of the slot $\mathcal{O}^{\varepsilon}$.

Theorem 3.4. For any $L>0$, there exists a positive constant $C(L)$ such that:

$$
\begin{gathered}
\left\|\widetilde{u}^{\varepsilon}-u^{\varepsilon}\right\|_{H^{1}\left(\mathcal{O}_{\varepsilon}^{L}\right)} \leq C(L) \varepsilon\|f\|_{L^{2}(\Omega)} . \\
\left\|\widetilde{u}^{\varepsilon}-u^{\varepsilon}\right\|_{L^{2}\left(\mathcal{O}_{\varepsilon}^{L}\right)} \leq C(L) \varepsilon^{\frac{3}{2}} \sqrt{|\log \varepsilon|}\|f\|_{L^{2}(\Omega)} .
\end{gathered}
$$

For any $L>L^{\prime}>0$, there exists a positive constant $C\left(L, L^{\prime}\right)$ such that:

$$
\left\|\widetilde{u}^{\varepsilon}-u^{\varepsilon}\right\|_{H^{1}\left(\mathcal{O}_{\varepsilon}^{L, L^{\prime}}\right)} \leq C\left(L, L^{\prime}\right) \varepsilon^{\frac{3}{2}} \sqrt{|\log \varepsilon|}\|f\|_{L^{2}(\Omega)} .
$$

In order to check that the above estimates are relevant, we have to compare the difference $u^{\varepsilon}-\widetilde{u}^{\varepsilon}$ to $u^{\varepsilon}$. For this we shall use the following inequalities (see Sect. 3.2):

$$
C_{1} \sqrt{\varepsilon}\left|u^{0}(0,0)\right|-C_{2} \varepsilon^{3 / 2} \sqrt{\log (\varepsilon)}\|f\|_{L^{2}(\Omega)} \leqslant\left\|u^{\varepsilon}\right\|_{H} \leqslant C_{3} \sqrt{\varepsilon}\|f\|_{L^{2}(\Omega)}
$$

which is valid for $H=H^{1}\left(\mathcal{O}_{\varepsilon}^{L}\right), L^{2}\left(\mathcal{O}_{\varepsilon}^{L}\right)$ and $H^{1}\left(\mathcal{O}_{\varepsilon}^{L, L^{\prime}}\right)$. In $(3.21), C_{1}, C_{2}$ and $C_{3}$ are three constants that only depend on the space $H$. This permits us to bound the relative errors:

$$
\frac{\left\|u^{\varepsilon}-\widetilde{u}^{\varepsilon}\right\|_{H}}{\left\|u^{\varepsilon}\right\|_{H}},
$$

as soon as $u_{0}(0,0) \neq 0$. The results are summarized in the following table.

\subsection{About the proof}

The study of the convergence of $u^{\varepsilon}$ to $u^{0}$ has an intrinsic interest in order to guarantee the relevance of our estimates on $u^{\varepsilon}-\widetilde{u}^{\varepsilon}$. A priori, looking at the convergence of $\widetilde{u}^{\varepsilon}$ to $u^{0}$ has no direct interest. However, we did not find how to study directly the error $u^{\varepsilon}-\widetilde{u}^{\varepsilon}$ without obtaining preliminary results on $\widetilde{u}^{\varepsilon}-u^{0}$. Indeed, the estimate (see Prop. 4.1)

$$
\left.\left\|\widetilde{u}^{\varepsilon}-u^{0}\right\|_{H^{1+s}\left(\Omega_{R}\right)} \leq C(R, s) \varepsilon^{1-s}\|f\|_{L^{2}(\Omega)}, \quad \forall s \in\right] 0,1[
$$


TABLE 2. Error estimates in the slot.

\begin{tabular}{|c|c|c|c|c|}
\hline$H$ & $=$ & $H^{1}\left(\mathcal{O}_{\varepsilon}^{L}\right)$ & $L^{2}\left(\mathcal{O}_{\varepsilon}^{L}\right)$ & $H^{1}\left(\mathcal{O}_{\varepsilon}^{L, L^{\prime}}\right)$ \\
\hline$\left\|u^{\varepsilon}-\widetilde{u}^{\varepsilon}\right\|_{H}$ & $\leqslant$ & $C \varepsilon$ & $C \varepsilon^{3 / 2}|\log (\varepsilon)|^{1 / 2}$ & $C \varepsilon^{3 / 2}|\log (\varepsilon)|^{1 / 2}$ \\
\hline$\frac{\left\|u^{\varepsilon}-\widetilde{u}^{\varepsilon}\right\|_{H}}{\left\|u^{\varepsilon}\right\|_{H}}$ & $\leqslant$ & $C \sqrt{\varepsilon}$ & $C \varepsilon|\log (\varepsilon)|^{1 / 2}$ & $C \varepsilon|\log (\varepsilon)|^{1 / 2}$ \\
\hline
\end{tabular}

will be crucial to obtain the estimate (3.11). Also, we have been able to obtain the optimality results of Theorem 3.2 only after having obtained the estimates of Theorem 3.3. That is why in the presentation of the proof of Theorems 3.1 to 3.4, we shall not follow the order we chose for their statement in Sections 3.1 and 3.2, but will proceed as indicated below:

(1) Study of the convergence of $u^{\varepsilon}$ to $u^{0}$ (Proof of Thms. 3.1). This is the object of Section 4.

(2) Estimates of $u^{\varepsilon}-\widetilde{u}^{\varepsilon}$ (Proof of Thms. 3.3 and 3.4). This is the object of Section 5.

(3) Proof of the optimality results (Thm. 3.2 and inequalities (3.21)). See both Sections 4 and 5.

\section{Convergence of $u^{\varepsilon}$ And $\widetilde{u}^{\varepsilon}$ TO The Limit half-SPACE Problem}

\subsection{Preliminary technical results}

In what follows, we shall naturally be led to identify $\Sigma_{\varepsilon}$ (see $(2.22)$ ) to the segment $] 0, \varepsilon[$ and $\partial \Omega$ to $\mathbb{R}$. Moreover, we shall denote by:

$$
\varphi: \Sigma_{\varepsilon} \mapsto \mathbb{C} \quad \longrightarrow \quad \tilde{\varphi}: \partial \Omega \mapsto \mathbb{C},
$$

the operator which consists in extending the function $\varphi$ to $\partial \Omega$ by 0 outside $\Sigma_{\varepsilon}$.

In the spaces $H^{s}(\partial \Omega) \equiv H^{s}(\mathbb{R})$, we shall work with the norm:

$$
\forall \varphi \in H^{s}(\mathbb{R}), \quad\|\varphi\|_{H^{s}(\mathbb{R})}^{2}=\int_{\mathbb{R}}\left(1+|k|^{2}\right)^{s}|\widehat{\varphi}(k)|^{2} \mathrm{~d} k,
$$

where $\widehat{\varphi}$ denotes the Fourier transform of $\varphi$.

Norms in $H^{s}\left(\Sigma_{\varepsilon}\right)$. In the rest of the paper, we shall have to work with Sobolev spaces on $\Sigma_{\varepsilon}$ (see (2.22)). Since this domain depends on $\epsilon$ and since we are looking for estimates in terms of $\varepsilon$, it is quite important to take care of the norms we shall use in the spaces $H^{s}\left(\Sigma_{\varepsilon}\right)$ and of the influence of this choice on the constants appearing in various inequalities in particular trace inequalities. The appropriate choice consists in using the basis $w_{n}^{\varepsilon}$ (see (2.35)) which is naturally associated with the problem.

Any function $\varphi \in L^{2}\left(\Sigma_{\varepsilon}\right) \equiv L^{2}(] 0, \varepsilon[)$ can be expanded as:

$$
\varphi=\sum_{n=0}^{+\infty} \varphi_{n}^{\varepsilon} w_{n}^{\varepsilon} \quad\left(\text { in } L^{2}\left(\Sigma_{\varepsilon}\right)\right), \quad \varphi_{n}^{\varepsilon}=\int_{\Sigma_{\varepsilon}} \varphi w_{n}^{\varepsilon} \mathrm{d} \sigma \equiv\left(\varphi, w_{n}^{\varepsilon}\right)_{\Sigma_{\varepsilon}}
$$

and one has:

$$
\|\varphi\|_{L^{2}\left(\Sigma_{\varepsilon}\right)}^{2}=\sum_{n=0}^{+\infty}\left|\varphi_{n}^{\varepsilon}\right|^{2}
$$

It is well known (see [29] for a similar proof) that one can also characterize the spaces $H^{s}\left(\Sigma_{\varepsilon}\right), 0 \leq s<3 / 2$ as:

$$
\varphi \in H^{s}\left(\Sigma_{\varepsilon}\right) \Longleftrightarrow\|\varphi\|_{H^{s}\left(\Sigma_{\varepsilon}\right)}^{2}=\sum_{n=0}^{+\infty}\left(1+\frac{n^{2} \pi^{2}}{\varepsilon^{2}}\right)^{s}\left|\varphi_{n}^{\varepsilon}\right|^{2}<+\infty .
$$


The dual spaces $H^{s}\left(\Sigma_{\varepsilon}\right)^{\prime}$ can be defined analogously but in the definition (4.2) of $\varphi_{n}^{\varepsilon}$, the scalar product in $L^{2}\left(\Sigma_{\varepsilon}\right)$ must be replaced by a duality bracket between $H^{s}\left(\Sigma_{\varepsilon}\right)^{\prime}$ and $H^{s}\left(\Sigma_{\varepsilon}\right)$.

The norm (4.4) is the one that we shall retain for the sequel. Note that these norms can be deduced one from the other by usual interpolation. Moreover, for $s=1$ we also have:

$$
\|\varphi\|_{H^{1}\left(\Sigma_{\varepsilon}\right)}^{2}=\int_{\Sigma_{\varepsilon}}\left(|\varphi|^{2}+\left|\varphi^{\prime}\right|^{2}\right) \mathrm{d} \sigma
$$

where $\varphi^{\prime}$ denotes the tangential derivative (y-derivative) of $\varphi$.

Trace inequalities. In this paragraph, we derive trace estimates in $H^{s}(\Omega)$.

Remark 4.1. The forthcoming results, namely the uniform inequality (4.8) and the Lemmas 4.1 and 4.3 , remain obviously valid if one replaces $\Omega$ by $\Omega_{R}$, for fixed $R>0$. It suffices to use a continuous extension operator from $H^{s}\left(\Omega_{R}\right)$ in $H^{s}(\Omega)$.

Since the $L^{2}$ and $H^{1}$ norms on $\Sigma_{\varepsilon}$ are defined as integrals, we have obviously:

$$
\left\{\begin{array}{l}
\forall \varphi \in L^{2}(\partial \Omega), \quad\|\varphi\|_{L^{2}\left(\Sigma_{\varepsilon}\right)} \leq\|\varphi\|_{L^{2}(\partial \Omega)} \\
\forall \varphi \in H^{1}(\partial \Omega), \quad\|\varphi\|_{H^{1}\left(\Sigma_{\varepsilon}\right)} \leq\|\varphi\|_{H^{1}(\partial \Omega)}
\end{array}\right.
$$

By interpolation we have:

$$
\forall \varphi \in H^{\frac{1}{2}}(\Omega), \quad\|\varphi\|_{H^{\frac{1}{2}}\left(\Sigma_{\varepsilon}\right)} \leq\|\varphi\|_{H^{\frac{1}{2}}(\partial \Omega)}
$$

Let $u$ be in $H^{1}(\Omega)$. For simplicity, we still denote by $u$ its trace on $\partial \Omega$ and use indifferently the notation $u_{n}^{\varepsilon}$ or $\left(u, w_{n}^{\varepsilon}\right)_{\Sigma_{\varepsilon}}$, the coefficients of the expansion in the basis $w_{n}^{\varepsilon}$ of the trace of $u$.

Using the trace theorem in $H^{1}(\Omega)$ (onto $H^{\frac{1}{2}}(\partial \Omega)$ ), we deduce from $(4.7)$ :

$$
\forall u \in H^{1}(\Omega), \quad\|u\|_{H^{\frac{1}{2}}\left(\Sigma_{\varepsilon}\right)} \leq C\|u\|_{H^{1}(\Omega)}
$$

Our purpose is to provide estimates of traces on $\Sigma_{\varepsilon}$ in function of $\varepsilon$. In our results, it is useful to distinguish the role of the coefficient $u_{0}^{\varepsilon}$ (on the first (constant) basis function $w_{0}^{\varepsilon}$ ) from the other coefficients $u_{n}^{\varepsilon}, n \geq 1$. Our first lemma concerns the first coefficient $u_{0}^{\varepsilon}$.

\section{Lemma 4.1.}

(i) For any $s \in] 0,1 / 2[$, there exists a constant $C(s)$ such that:

$$
\forall u \in H^{\frac{1}{2}+s}(\Omega), \quad\left|u_{0}^{\varepsilon}\right| \leqslant C(s) \varepsilon^{s}\|u\|_{H^{\frac{1}{2}+s}(\Omega)} .
$$

(ii) There exists a constant $C$ such that:

$$
\forall u \in H^{1}(\Omega), \quad\left|u_{0}^{\varepsilon}\right| \leqslant C \sqrt{\varepsilon|\log (\varepsilon)|}\|u\|_{H^{1}(\Omega)} .
$$

(iii) For any $s \in] 0,+\infty[$, there exists a constant $C(s)$ such that:

$$
\forall u \in H^{1+s}(\Omega), \quad\left|u_{0}^{\varepsilon}\right| \leqslant C(s) \sqrt{\varepsilon}\|u\|_{H^{1+s}(\Omega)} .
$$

Proof. By definition of $u_{0}^{\varepsilon}$, if we identify $\partial \Omega$ to $\mathbb{R}$, we can write:

$$
\left|u_{0}^{\varepsilon}\right|=\left|\int_{\mathbb{R}} u(0, y) \tilde{w}_{0}^{\varepsilon}(y) \mathrm{d} y\right| \leqslant\|\tilde{u}\|_{H^{s}(\mathbb{R})} \times\left\|\tilde{w}_{0}^{\varepsilon}\right\|_{H^{-s}(\mathbb{R})}
$$


where $\tilde{w}_{0}^{\varepsilon}$ is the extension of $w_{0}^{\varepsilon}$ by 0 outside $\Sigma_{\varepsilon}$. Consequently, using trace theorem, we get:

$$
\left|u_{0}^{\varepsilon}\right| \leqslant C(s)\|u\|_{H^{1 / 2+s}(\Omega)} \times\left\|\tilde{w}_{0}^{\varepsilon}\right\|_{H^{-s}(\mathbb{R})} .
$$

The result is then a direct consequence of Lemma 4.2 below.

Lemma 4.2. When $\varepsilon \rightarrow 0$, one has the following results:

$$
\begin{gathered}
\left\|\widetilde{w}_{0}^{\varepsilon}\right\|_{H^{s}(\mathbb{R})} \sim C(s) \varepsilon^{-s} \quad \text { for }-1 / 2<s<1 / 2, \\
\left\|\widetilde{w}_{0}^{\varepsilon}\right\|_{H^{-\frac{1}{2}(\mathbb{R})}} \sim \sqrt{2 \varepsilon|\log (\varepsilon)|} \\
\left\|\tilde{w}_{0}^{\varepsilon}\right\|_{H^{s}(\mathbb{R})} \sim C(s) \sqrt{\varepsilon} \quad \text { for } s<-\frac{1}{2}
\end{gathered}
$$

where

$$
\begin{cases}C(s)=\left(\int_{\mathbb{R}} t^{2(s-1)} \sin ^{2}(t) \mathrm{d} t\right)^{\frac{1}{2}}, & \text { for }-\frac{1}{2}<s<\frac{1}{2}, \\ C(s)=\left(\int_{\mathbb{R}}\left(1+t^{2}\right)^{s} \mathrm{~d} t\right)^{\frac{1}{2}}, & \text { for } s<-\frac{1}{2} .\end{cases}
$$

Proof. We only give here the proof of (4.15). The Fourier transform of $\tilde{w}_{0}^{\varepsilon}$ is given by:

$$
\widehat{w}_{0}^{\varepsilon}(k)=\sqrt{\varepsilon} \operatorname{sinc}\left(\frac{k \varepsilon}{2}\right), \quad \text { where } \operatorname{sinc}(x)=\frac{\sin x}{x} .
$$

Consequently, $\left\|\widetilde{w}_{0}^{\varepsilon}\right\|_{H^{-\frac{1}{2}}(\mathbb{R})}^{2}=\varepsilon \int_{\mathbb{R}} \frac{\operatorname{sinc}^{2}\left(\frac{k \varepsilon}{2}\right)}{\left(1+k^{2}\right)^{\frac{1}{2}}} \mathrm{~d} k=\varepsilon \int_{\mathbb{R}} \frac{\operatorname{sinc}^{2}(t)}{\left(\left(\frac{\varepsilon}{2}\right)^{2}+t^{2}\right)^{\frac{1}{2}}} \mathrm{~d} t$.

We split the last integral into three parts:

$$
\left\|\widetilde{w}_{0}^{\varepsilon}\right\|_{H^{-\frac{1}{2}(\mathbb{R})}}^{2}=\varepsilon\left[\int_{-1}^{1} \frac{1}{\left(\left(\frac{\varepsilon}{2}\right)^{2}+t^{2}\right)^{\frac{1}{2}}} \mathrm{~d} t+\int_{|t|>1} \frac{\operatorname{sinc}^{2}(t)}{\left(\left(\frac{\varepsilon}{2}\right)^{2}+t^{2}\right)^{\frac{1}{2}}} \mathrm{~d} t+\int_{-1}^{1} \frac{\operatorname{sinc}^{2}(t)-1}{\left(\left(\frac{\varepsilon}{2}\right)^{2}+t^{2}\right)^{\frac{1}{2}}} \mathrm{~d} t\right] .
$$

The first term can be computed explicitly:

$$
\int_{-1}^{1} \frac{1}{\left(\left(\frac{\varepsilon}{2}\right)^{2}+t^{2}\right)^{\frac{1}{2}}} \mathrm{~d} t=2 \operatorname{argsh}\left(\frac{2}{\varepsilon}\right) \sim 2|\log \varepsilon| \quad(\varepsilon \rightarrow 0) .
$$

The inequality $\frac{\operatorname{sinc}^{2}(t)}{\left(\left(\frac{\varepsilon}{2}\right)^{2}+t^{2}\right)^{\frac{1}{2}}} \leqslant \frac{1}{|t|^{3}}$ and permits us to use Lebesgue's theorem to show that:

$$
\lim _{\varepsilon \rightarrow 0} \int_{|t|>1} \frac{\operatorname{sinc}^{2}(t)}{\left(\left(\frac{\varepsilon}{2}\right)^{2}+t^{2}\right)^{\frac{1}{2}}} \mathrm{~d} t=\int_{|t|>1} \frac{\operatorname{sinc}^{2}(t)}{|t|} \mathrm{d} t
$$

In the same way, the bound $\frac{\left|\operatorname{sinc}^{2}(t)-1\right|}{\left(\left(\frac{\varepsilon}{2}\right)^{2}+t^{2}\right)^{\frac{1}{2}}} \leqslant \frac{\left|\operatorname{sinc}^{2}(t)-1\right|}{|t|}$ permits us to obtain:

$$
\lim _{\varepsilon \rightarrow 0} \int_{-1}^{1} \frac{\operatorname{sinc}^{2}(t)-1}{\left(\left(\frac{\varepsilon}{2}\right)^{2}+t^{2}\right)^{\frac{1}{2}}} \mathrm{~d} t=\int_{-1}^{1} \frac{\operatorname{sinc}^{2}(t)-1}{|t|} \mathrm{d} t .
$$

The conclusion follows immediately. 
Next we introduce the following semi-norm in $H^{s}\left(\Sigma_{\varepsilon}\right)$ :

$$
\|\varphi\|_{H_{*}^{s}\left(\Sigma_{\varepsilon}\right)}^{2}=\sum_{n=1}^{+\infty}\left(1+\frac{n^{2} \pi^{2}}{\varepsilon^{2}}\right)^{s}\left|\varphi_{n}^{\varepsilon}\right|^{2}
$$

\section{Lemma 4.3.}

(i) For any $s \in[0,1[$, there exists a constant $C(s)$ such that:

$$
\forall u \in H^{1+s}(\Omega), \quad\|u\|_{H_{*}^{1 / 2}\left(\Sigma_{\varepsilon}\right)} \leqslant C(s) \varepsilon^{s}\|u\|_{H^{1+s}(\Omega)} .
$$

(ii) There exists a constant $C$ such that:

$$
\forall u \in H^{2}(\Omega), \quad\|u\|_{H_{*}^{1 / 2}\left(\Sigma_{\varepsilon}\right)} \leqslant C \varepsilon \sqrt{|\log (\varepsilon)|}\|u\|_{H^{2}(\Omega)} .
$$

(iii) For any $s \in] 1,+\infty[$, there exists a constant $C(s)$ such that:

$$
\forall u \in H^{1+s}(\Omega), \quad\|u\|_{H_{*}^{1 / 2}\left(\Sigma_{\varepsilon}\right)} \leqslant C(s) \varepsilon\|u\|_{H^{1+s}(\Omega)} .
$$

Proof. (i) We first prove (4.22) for $s \leqslant 1 / 2$. Let $P^{\varepsilon}$ be the orthogonal projection from $L^{2}\left(\Sigma_{\varepsilon}\right)$ on the subspace of constant functions (generated by $w_{0}^{\varepsilon}$ ). As a direct consequence of Bramble-Hilbert lemma, we have:

$$
\begin{cases}\left\|u-P^{\varepsilon} u\right\|_{L^{2}\left(\Sigma_{\varepsilon}\right)} \leqslant C \varepsilon\left\|u^{\prime}\right\|_{L^{2}\left(\Sigma_{\varepsilon}\right)}, & \forall u \in H^{1}\left(\Sigma_{\varepsilon}\right), \\ \left\|u-P^{\varepsilon} u\right\|_{H^{1}\left(\Sigma_{\varepsilon}\right)} \leqslant C\left\|u^{\prime}\right\|_{L^{2}\left(\Sigma_{\varepsilon}\right)}, & \forall u \in H^{1}\left(\Sigma_{\varepsilon}\right),\end{cases}
$$

and by interpolation

$$
\|u\|_{H_{*}^{1 / 2}\left(\Sigma_{\varepsilon}\right)}=\left\|u-P^{\varepsilon} u\right\|_{H^{1 / 2}\left(\Sigma_{\varepsilon}\right)} \leqslant C \sqrt{\varepsilon}\left\|u^{\prime}\right\|_{L^{2}\left(\Sigma_{\varepsilon}\right)}
$$

which shows the result for $s=1 / 2$ since, by usual trace theorem:

$$
\left\|u^{\prime}\right\|_{L^{2}\left(\Sigma_{\varepsilon}\right)} \leq\|u\|_{H^{1}\left(\Sigma_{\varepsilon}\right)} \leq C\|u\|_{H^{3 / 2}(\Omega)} .
$$

By interpolation with (4.8), we get the result for $0<s<1 / 2$.

For $s>1 / 2$ we shall use the following result (see [29], Thm. 11.2 p. 63) for Sobolev spaces in one dimension:

For any $r \in[0,1 / 2[$ there exists $C(r)>0$ such that:

$$
\forall v(y) \in H^{r}\left(\mathbb{R}^{+}\right), \quad v / y^{r} \in L^{2}\left(\mathbb{R}^{+}\right), \quad\left\|\frac{v}{y^{r}}\right\|_{L^{2}\left(\mathbb{R}^{+}\right)} \leqslant C(r)\|v\|_{H^{r}\left(\mathbb{R}^{+}\right)} .
$$

Let us apply (4.28) to $v=u^{\prime}$, the $y$-derivative of the trace on $\partial \Omega$ of $u \in H^{\frac{3}{2}+r}(\Omega)$ :

$$
\left\|u^{\prime}\right\|_{L^{2}\left(\Sigma_{\varepsilon}\right)}=\left\|\frac{y^{r}}{y^{r}} u^{\prime}\right\|_{L^{2}\left(\Sigma_{\varepsilon}\right)} \leqslant \varepsilon^{r}\left\|\frac{u^{\prime}}{y^{r}}\right\|_{L^{2}\left(\Sigma_{\varepsilon}\right)} \leqslant \varepsilon^{r}\left\|\frac{u^{\prime}}{y^{r}}\right\|_{L^{2}\left(\mathbb{R}^{+}\right)},
$$

where we have identified the part of $\partial \Omega$ made of points $(0, y), y>0$ to $\mathbb{R}^{+}$.

Therefore, combining (4.26) and (4.28) with (4.29) and trace theorem in $H^{\frac{3}{2}+r}(\Omega)$, we get:

$$
\|u\|_{H_{*}^{1 / 2}\left(\Sigma_{\varepsilon}\right)} \leqslant C(r) \varepsilon^{1 / 2+r}\left\|u^{\prime}\right\|_{H^{r}\left(\mathbb{R}^{+}\right)} \leqslant C(r) \varepsilon^{1 / 2+r}\|\tilde{u}\|_{H^{\frac{3}{2}+r}(\Omega)}
$$


which is nothing but (4.22) for $1 / 2<s<1$ ( $\operatorname{set} r=s-1 / 2$ ).

(ii) For any $v$ in $H^{1}(\Omega)$ we have:

$$
\|v\|_{L^{2}\left(\Sigma_{\varepsilon}\right)}=\left|v_{0}^{\varepsilon}\right|^{2}+\left\|v-P^{\varepsilon} v\right\|_{L^{2}\left(\Sigma_{\varepsilon}\right)}^{2} .
$$

By interpolation between the first inequality of (4.25) and

$$
\left\|v-P^{\varepsilon} v\right\|_{L^{2}\left(\Sigma_{\varepsilon}\right)} \leqslant\|v\|_{L^{2}\left(\Sigma_{\varepsilon}\right)}
$$

we get:

$$
\left\|v-P^{\varepsilon} v\right\|_{L^{2}\left(\Sigma_{\varepsilon}\right)} \leqslant C \sqrt{\varepsilon}\|v\|_{H^{1 / 2}\left(\Sigma_{\varepsilon}\right)} .
$$

It suffices to use (4.8) and (4.10) to obtain

$$
\|v\|_{L^{2}\left(\Sigma_{\varepsilon}\right)} \leqslant C \sqrt{\varepsilon|\log (\varepsilon)|}\|v\|_{H^{1}(\Omega)} .
$$

Thus, applying (4.34) to (4.26), with $v=u^{\prime}$, we obtain (4.23).

(iii) In dimension 2 , for any $s>1, H^{1+s}(\Omega)$ is continuously embedded in $W^{1, \infty}(\bar{\Omega})$ :

$$
\forall u \in H^{1+s}(\Omega), \quad\|\nabla u\|_{L^{\infty}(\bar{\Omega})} \leqslant C(s)\|u\|_{H^{1+s}(\Omega)} .
$$

Thus, coming back to (4.26), we get:

$$
\|u\|_{H_{*}^{1 / 2}\left(\Sigma_{\varepsilon}\right)} \leq C \sqrt{\varepsilon}\left\|u^{\prime}\right\|_{L^{2}\left(\Sigma_{\varepsilon}\right)} \leq C \varepsilon\left\|u^{\prime}\right\|_{L^{\infty}\left(\Sigma_{\varepsilon}\right)} \leq C(s) \varepsilon\|u\|_{H^{1+s}(\Omega)},
$$

which concludes the proof.

\subsection{Study of $u^{\varepsilon}-u^{0}$. Proof of Theorem 3.1}

Reformulation of problem $(2.10,2.11)$ in $\Omega$ As $u^{0}$ is only defined in $\Omega$, it is useful to characterize the restriction of $u^{\varepsilon}$ to $\Omega$. This is done easily by deriving an exact Dirichlet-to-Neumann boundary condition for $u^{\varepsilon}$ on $\Sigma_{\varepsilon}$ (see (2.22)) from the general form (2.39) of the solution inside the slot [21]. Proceeding this way, one easily shows that the restriction of $u^{\varepsilon}$ to $\Omega$ is characterized as the unique solution of the boundary value problem:

where the operator:

$$
\begin{cases}-\Delta u^{\varepsilon}-\omega^{2} u^{\varepsilon}=f, & \text { in } \Omega, \\ \frac{\partial u^{\varepsilon}}{\partial x}=0 & \text { on } \partial \Omega \backslash \Sigma_{\varepsilon} \\ \left.\frac{\partial u^{\varepsilon}}{\partial x}\right|_{\Sigma_{\varepsilon}}+T^{\varepsilon}\left(\left.u^{\varepsilon}\right|_{\Sigma_{\varepsilon}}\right)=0, & \text { on } \Sigma_{\varepsilon}, \\ u^{\varepsilon} \text { is outgoing at infinity, } & \text { (see Rem. 2.3), }\end{cases}
$$

is defined by (the numbers $\xi_{n}^{\varepsilon}(\omega)$ are given by $(2.38)$ ):

$$
T^{\varepsilon}: \varphi=\sum_{n=0}^{+\infty} \varphi_{n}^{\varepsilon} w_{n}^{\varepsilon} \longrightarrow T^{\varepsilon} \varphi=-i \omega \varphi_{0}^{\varepsilon} w_{0}^{\varepsilon}+\sum_{n=1}^{+\infty} \xi_{n}^{\varepsilon}(\omega) \varphi_{n}^{\varepsilon} w_{n}^{\varepsilon} .
$$


Using the fact that the radiation condition at infinity can be written on $\Gamma_{R}$ for $R$ large enough (cf. Sect. 2), we can also characterize the restriction of $u^{\varepsilon}$ to $\Omega_{R}$ as the unique solution of the variational problem:

$$
\left\{\begin{array}{l}
\text { Find } u^{\varepsilon} \in H^{1}\left(\Omega_{R}\right) \text { such that: } \\
a_{R}\left(u^{\varepsilon}, v\right)+b_{R}\left(u^{\varepsilon}, v\right)+b^{\varepsilon}\left(u^{\varepsilon}, v\right)=\int_{\Omega_{R}} f \bar{v} \mathrm{~d} \mathbf{x}, \quad \forall v \in H^{1}\left(\Omega_{R}\right),
\end{array}\right.
$$

where the bilinear forms $a(.,$.$) and b_{R}(.,$.$) are respectively defined by (2.28)$ (in the case $\left.\Omega_{R}^{\prime}=\emptyset\right)$ and $(2.29)$, while $b^{\varepsilon}(.,$.$) is defined by:$

$$
b^{\varepsilon}(u, v)=-i \omega u_{0}^{\varepsilon} \overline{v_{0}^{\varepsilon}}+\sum_{n=1}^{+\infty} \xi_{n}^{\varepsilon}(\omega) u_{n}^{\varepsilon} \overline{v_{n}^{\varepsilon}}, \quad \forall(u, v) \in\left[H^{1}\left(\Omega_{R}\right)\right]^{2}
$$

Thanks to Riesz theorem,we can define two linear operators:

$$
A_{R}, B^{\varepsilon} \in \mathcal{L}\left(H^{1}\left(\Omega_{R}\right), H^{1}\left(\Omega_{R}\right)\right)
$$

such that:

$$
\left\{\begin{array}{l}
\left(A_{R} u, v\right)_{H^{1}\left(\Omega_{R}\right)}=a_{R}(u, v)+b_{R}(u, v), \quad \forall(u, v) \in\left[H^{1}\left(\Omega_{R}\right)\right]^{2}, \\
\left(B^{\varepsilon} u, v\right)_{H^{1}\left(\Omega_{R}\right)}=b^{\varepsilon}(u, v), \quad \forall(u, v) \in\left[H^{1}\left(\Omega_{R}\right)\right]^{2}
\end{array}\right.
$$

and $\varphi_{f} \in H^{1}(\Omega)$ such that:

$$
\left(\varphi_{f}, v\right)_{H^{1}\left(\Omega_{R}\right)}=\int_{\Omega_{R}} f \bar{v} \mathrm{~d} \mathbf{x} .
$$

The problem satisfied by the restriction of $u^{\varepsilon}$ to $\Omega_{R}$ can thus be written in a compact way:

$$
\text { Find } u^{\varepsilon} \in H^{1}\left(\Omega_{R}\right) \text { such that } \quad\left(A_{R}+B^{\varepsilon}\right) u^{\varepsilon}=\varphi_{f} \text {. }
$$

Note that, in the same way, the restriction of $u^{0}$ to $\Omega_{R}$ is the unique solution of:

$$
\text { Find } u^{0} \in H^{1}\left(\Omega_{R}\right) \text { such that } \quad A_{R} u^{0}=\varphi_{f} \text {. }
$$

Note that the well posedness of problems (2.42) and (2.45) means that $A_{R}$ and $A_{R}+B^{\varepsilon}$ are both isomorphisms. Moreover, the error $u^{\varepsilon}-u^{0}$ satisfies in $\Omega_{R}$ :

$$
\left(A_{R}+B^{\varepsilon}\right)\left(u^{0}-u^{\varepsilon}\right)=B^{\varepsilon} u^{0}
$$

In the rest of this section, we shall fix $R$ (large enough in order that the support of $f$ be included in $\Omega_{R}$ ). In our estimates, for simplicity, we shall not mention explicitly the dependence of the various constants with respect to $R$. These constants will de denoted by $C$ and, as usual, the value of $C$ may change from one line to another. We shall keep this principle throughout the rest of the article.

Stability result. The convergence proof of $u^{\varepsilon}$ to $u^{0}$ will use a stability result. i.e. a uniform estimate of $\left(A_{R}+B^{\varepsilon}\right)^{-1}$ in the operator norm.

Lemma 4.4. There exists a positive constant $C$ such that:

$$
\left\|\left(A_{R}+B^{\varepsilon}\right)^{-1}\right\|_{\mathcal{L}\left(H^{1}\left(\Omega_{R}\right)\right)} \leq C .
$$


Proof. We make a proof by contradiction. If (4.48) were false, there would exist a sequence $v^{\varepsilon}, \varepsilon \rightarrow 0$ such that:

$$
\left\|v^{\varepsilon}\right\|_{H^{1}\left(\Omega_{R}\right)}=1 \quad \text { and } \quad \varphi^{\varepsilon}=\left(A_{R}+B^{\varepsilon}\right) v^{\varepsilon} \rightarrow 0 \quad \text { in } H^{1}\left(\Omega_{R}\right) .
$$

By compactness, let us extract from $v^{\varepsilon}$ a subsequence (still denoted $v^{\varepsilon}$ ) with $\varepsilon \rightarrow 0$ such that:

$$
v^{\varepsilon} \rightarrow v^{0} \quad \text { weakly in } H^{1}\left(\Omega_{R}\right) \text { and strongly in } L^{2}\left(\Omega_{R}\right) .
$$

By definition of $A_{R}$ and $B^{\varepsilon}$, we have:

$$
\forall v \in H^{1}\left(\Omega_{R}\right), \quad a_{R}\left(v^{\varepsilon}, v\right)+b_{R}\left(v^{\varepsilon}, v\right)+b^{\varepsilon}\left(v^{\varepsilon}, v\right)=\left(\varphi^{\varepsilon}, v\right) .
$$

Let us introduce the subspace of $H^{1}\left(\Omega_{R}\right)$ defined by $(B(0, r)$ denoting, for any $r>0$, the ball of center 0 and radius $r$ ):

$$
H_{00}^{1}\left(\Omega_{R}\right) \equiv\left\{v \in H^{1}\left(\Omega_{R}\right) / \exists r=r^{*}(v)>0 / v=0 \text { in } \Omega_{R} \cap B(0, r)\right\} .
$$

Obviously, for any $v \in H_{00}^{1}\left(\Omega_{R}\right), \quad b^{\varepsilon}\left(v^{\varepsilon}, v\right)=0$ pour $\varepsilon<r^{*}(v)$, which implies that (we use the weak convergence of $v^{\varepsilon}$ in $\left.H^{1}\left(\Omega_{R}\right)\right)$ :

$$
\forall v \in H_{00}^{1}\left(\Omega_{R}\right), \quad \lim _{\varepsilon \rightarrow 0} a_{R}\left(v^{\varepsilon}, v\right)+b_{R}\left(v^{\varepsilon}, v\right)+b^{\varepsilon}\left(v^{\varepsilon}, v\right)=a\left(v^{0}, v\right) .
$$

Taking the limit of (4.51) when $\varepsilon \rightarrow 0$, we get:

$$
a_{R}\left(v_{0}, v\right)+b_{R}\left(v_{0}, v\right)=0, \quad \forall v \in H_{00}^{1}\left(\Omega_{R}\right) .
$$

It is well known (see [24] for an explicit proof in $2 \mathrm{D}$ ) that $H_{00}^{1}\left(\Omega_{R}\right)$ is dense in $H^{1}\left(\Omega_{R}\right)$. Thus, by continuity of $a_{R}(.,$.$) and b_{R}(.,$.$) , we also get:$

$$
a_{R}\left(v_{0}, v\right)+b_{R}\left(v_{0}, v\right)=0, \quad \forall v \in H^{1}\left(\Omega_{R}\right),
$$

that is to say $A_{R} v^{0}=0$ and therefore $v^{0}=0$ since $A_{R}$ is injective.

Let us take $v=v^{\varepsilon}$ in (4.51) and look at the real part of the resulting equality:

$$
\int_{\Omega_{R}}\left|\nabla v^{\varepsilon}\right|^{2} \mathrm{~d} x+\sum_{n=1}^{+\infty} \xi_{n}^{\varepsilon}(\omega)\left|\left(v^{\varepsilon}\right)_{n}^{\varepsilon}\right|^{2}+\mathcal{R} e b_{R}\left(v^{\varepsilon}, v^{\varepsilon}\right)=\mathcal{R} e\left(\varphi^{\varepsilon}, v^{\varepsilon}\right)_{H^{1}\left(\Omega_{R}\right)}+\omega^{2} \int_{\Omega_{R}}\left|v^{\varepsilon}\right|^{2} \mathrm{~d} x
$$

From (4.49), (2.30) and the positivity of the coefficients $\xi_{n}^{\varepsilon}(\omega)$, we deduce that:

$$
\left\|\nabla v^{\varepsilon}\right\|_{L^{2}\left(\Omega_{R}\right)}^{2} \leq\left\|\varphi^{\varepsilon}\right\|_{H^{1}\left(\Omega_{R}\right)}+\omega^{2}\left\|v^{\varepsilon}\right\|_{L^{2}\left(\Omega_{R}\right)}^{2},
$$

which, combined with the facts that $\varphi^{\varepsilon}$ and $v^{\varepsilon}$ converge to 0 respectively in $H^{1}\left(\Omega_{R}\right)$ and $L^{2}\left(\Omega_{R}\right)$, proves that $v^{\varepsilon} \rightarrow 0$ strongly in $H^{1}\left(\Omega_{R}\right)$. This contradicts $\left\|v^{\varepsilon}\right\|_{H^{1}\left(\Omega_{R}\right)}=1$.

Consistency estimate. According to equation (4.47), to complete the error analysis, we have to estimate $B^{\varepsilon} u^{0}$ in $H^{1}\left(\Omega_{R}\right)$. It is easy to see that $B^{\varepsilon}$ is uniformly bounded in $\mathcal{L}\left(H^{1}\left(\Omega_{R}\right)\right)$. This is a direct consequence of the uniform trace estimate (4.8). However, it can also be shown that $B^{\varepsilon}$ does not tend to 0 in $\mathcal{L}\left(H^{1}\left(\Omega_{R}\right)\right)$ when $\varepsilon$ tends to 0: we refer the reader to [24] for the construction of a sequence $v^{\varepsilon}$ in $H^{1}\left(\Omega_{R}\right)$ such that $b_{\varepsilon}\left(v^{\varepsilon}, v^{\varepsilon}\right) /\left\|v^{\varepsilon}\right\|_{H^{1}\left(\Omega_{R}\right)}^{2} \geq \alpha>0$ ( $\alpha$ independent of $\varepsilon$. However, we are going to see that, due to the fact that $u^{0}$ is independent of $\varepsilon$ and sufficiently smooth, $B^{\varepsilon} u^{0}$ does tend to 0 in $H^{1}\left(\Omega_{R}\right)$.

The proof uses a decomposition of the operator $B^{\varepsilon}$ in the form:

$$
B^{\varepsilon}=B_{0}^{\varepsilon}+R^{\varepsilon},
$$


that corresponds, in an obvious manner (cf. (4.43)), to the decomposition of $b^{\varepsilon}(u, v)$ as:

$$
\left\{\begin{array}{l}
b^{\varepsilon}(u, v)=b_{0}^{\varepsilon}(u, v)+r^{\varepsilon}(u, v) \\
b_{0}^{\varepsilon}(u, v)=-i \omega u_{0}^{\varepsilon} \overline{v_{0}^{\varepsilon}}, \quad r^{\varepsilon}(u, v)=\sum_{n=1}^{+\infty} \xi_{n}^{\varepsilon}(\omega) u_{n}^{\varepsilon} \overline{v_{n}^{\varepsilon}}
\end{array}\right.
$$

Lemma 4.5. There exists a constant $C>0$ such that:

$$
\begin{gathered}
\left\|B_{0}^{\varepsilon} u^{0}\right\|_{H^{1}\left(\Omega_{R}\right)} \leqslant C \varepsilon \sqrt{|\log \varepsilon|}\|f\|_{L^{2}(\Omega)}, \\
\left\|R^{\varepsilon} u^{0}\right\|_{H^{1}\left(\Omega_{R}\right)} \leqslant C \varepsilon\|f\|_{L^{2}(\Omega)} .
\end{gathered}
$$

Proof. We first prove (4.59). By definition of $B_{0}^{\varepsilon}$, for any $u \in H^{1}\left(\Omega_{R}\right)$ :

$$
\left\|B_{0}^{\varepsilon} u\right\|_{H^{1}\left(\Omega_{R}\right)}=\sup _{v \in H^{1}\left(\Omega_{R}\right)} \frac{b_{0}^{\varepsilon}(u, v)}{\|v\|_{H^{1}\left(\Omega_{R}\right)}} \leqslant C\left|u_{0}^{\varepsilon}\right| \sup _{v \in H^{1}\left(\Omega_{R}\right)} \frac{\left|v_{0}^{\varepsilon}\right|}{\|v\|_{H^{1}\left(\Omega_{R}\right)}} .
$$

Using (4.10) - see Lemma 4.1 and Remark 4.1 - we thus get:

$$
\left\|B_{0}^{\varepsilon} u\right\|_{H^{1}\left(\Omega_{R}\right)} \leqslant C \sqrt{\varepsilon|\log (\varepsilon)|}\left|u_{0}^{\varepsilon}\right| .
$$

If moreover $u \in H^{1+s}\left(\Omega_{R}\right), s>0$, we deduce from Lemma 4.1, estimate (4.11), that:

$$
\left\|B_{0}^{\varepsilon} u\right\|_{H^{1}(\Omega)} \leqslant C \varepsilon \sqrt{|\log (\varepsilon)|}\|u\|_{H^{1+s}\left(\Omega_{R}\right)} .
$$

To obtain (4.59), it suffices to apply (4.63) to $u=u^{0}$ and $s=1$ which satisfies $u \in H^{2}\left(\Omega_{R}\right)$ and $\|u\|_{H^{1+s}\left(\Omega_{R}\right)} \leq$ $C\|f\|_{L^{2}(\Omega)}$.

Next, we prove (4.60). By definition of $R^{\varepsilon}$, we have, for any $u \in H^{1}\left(\Omega_{R}\right)$,

$$
\left\|R^{\varepsilon} u\right\|_{H^{1}(\Omega)}=\sup _{v \in H^{1}\left(\Omega_{R}\right)} \frac{r^{\varepsilon}(u, v)}{\|v\|_{H^{1}\left(\Omega_{R}\right)}} .
$$

Using the inequality $\left|\xi_{n}^{\varepsilon}(\omega)\right| \leqslant\left(1+\pi^{2} n^{2} / \varepsilon^{2}\right)^{\frac{1}{2}}$ and the definition (4.58) of $r^{\varepsilon}(.,$.$) we get:$

$$
r^{\varepsilon}(u, v) \leq\|u\|_{H_{*}^{\frac{1}{2}}\left(\Sigma_{\varepsilon}\right)}\|v\|_{H_{*}^{\frac{1}{2}}\left(\Sigma_{\varepsilon}\right)} .
$$

Using (4.22) - see Lemma 4.3 and Remark 4.1 - with $s=0$, we get:

$$
\left\|R^{\varepsilon} u\right\|_{H^{1}\left(\Omega_{R}\right)} \leq C\|u\|_{H_{*}^{\frac{1}{2}}\left(\Sigma_{\varepsilon}\right)} .
$$

As $f$ is compactly supported in $\Omega$, we know that, if $\mathcal{V}$ denotes a bounded neighborhood of the origin which does not intersect the support of $f$, then:

$$
\forall s>1, \quad u^{0} \in H^{1+s}(\mathcal{V}), \text { and } \quad\left\|u^{0}\right\|_{H^{1+s}(\mathcal{V})} \leq C(s, \mathcal{V})\|f\|_{L^{2}(\Omega)}
$$

So, by Lemma 4.3, equation (4.24) (applied for instance with $s=2$ ! - see also Rem. 4.1), we get:

$$
\left\|u^{0}\right\|_{H_{*}^{\frac{1}{2}}\left(\Sigma_{\varepsilon}\right)} \leq C \varepsilon\|f\|_{L^{2}(\Omega)}
$$

we deduce (4.60) from (4.66) and (4.68). 
Remark 4.2. The inequality (4.62), joined to (4.10), proves that, contrary to $B^{\varepsilon}, B_{0}^{\varepsilon}$ tends to 0 with $\varepsilon$ in $\mathcal{L}\left(H^{1}\left(\Omega_{R}\right)\right)$ :

$$
\left\|B_{0}^{\varepsilon}\right\|_{\mathcal{L}\left(H^{1}\left(\Omega_{R}\right)\right)} \leq C \varepsilon|\log (\varepsilon)| .
$$

Remark 4.3. If the support of $f$ contains the origin, (4.67) is true only for $0 \leq s \leq 1$. This time, we can apply (4.23) to obtain:

$$
\left\|R^{\varepsilon} u^{0}\right\|_{H^{1}\left(\Omega_{R}\right)} \leq C \varepsilon \sqrt{|\log (\varepsilon)|}\|f\|_{L^{2}(\Omega)} .
$$

$H^{1}\left(\Omega_{R}\right)$-estimate of $u^{\varepsilon}-u_{0}$. We simply complete the proof of (3.5). From equation (4.47) and Lemma 4.4, we deduce:

$$
\left\|u^{\varepsilon}-u^{0}\right\|_{H^{1}\left(\Omega_{R}\right)} \leq\left\|B^{\varepsilon} u^{0}\right\|_{H^{1}\left(\Omega_{R}\right)} \leq\left\|B_{0}^{\varepsilon} u^{0}\right\|_{H^{1}\left(\Omega_{R}\right)}+\left\|R^{\varepsilon} u^{0}\right\|_{H^{1}\left(\Omega_{R}\right)} .
$$

Then, it suffices to invoke Lemma 4.5 to conclude.

Remark 4.4. According to Remark 4.3, if the support of $f$ contains the origin, the error estimate (3.5) is, a priori, no longer true. However, one has:

$$
\left\|u^{\varepsilon}-u^{0}\right\|_{H^{1}\left(\Omega_{R}\right)} \leq C \varepsilon \sqrt{|\log (\varepsilon)|}\|f\|_{L^{2}(\Omega)} .
$$

$L^{2}\left(\Omega_{R}\right)$-estimate of $u^{\varepsilon}-u_{0}$. Estimate (3.6) could be proven directly using a duality method as we shall do for $\widetilde{u}^{\varepsilon}-u^{0}$ in Section (4.3). However, it will also appear as a direct consequence of forthcoming results (see the last paragraph of Sect. 4).

\subsection{Study of $\widetilde{u}^{\varepsilon}-u^{0}$}

Statement of the approximation results. We give below approximation results for $\widetilde{u}^{\varepsilon}-u^{0}$ which are analogous to those of theorems 3.1 and 3.2 for $u^{\varepsilon}-u^{0}$.

Proposition 4.1. For any $s \in\left[0,1\left[\right.\right.$, the function $\widetilde{u}^{\varepsilon}$ converges in $H^{1+s}\left(\Omega_{R}\right)$ to $u^{0}$ when $\varepsilon$ tends to 0 and one has the error estimates:

$$
\begin{gathered}
\left.\left\|\widetilde{u}^{\varepsilon}-u^{0}\right\|_{H^{1+s}\left(\Omega_{R}\right)} \leq C(R, s) \varepsilon^{1-s}\|f\|_{L^{2}(\Omega)}, \quad \forall s \in\right] 0,1[, \\
\left\|\widetilde{u}^{\varepsilon}-u^{0}\right\|_{H^{1}\left(\Omega_{R}\right)} \leq C(R) \varepsilon \sqrt{|\log (\varepsilon)|}\|f\|_{L^{2}(\Omega)}, \\
\left\|\widetilde{u}^{\varepsilon}-u^{0}\right\|_{L^{2}\left(\Omega_{R}\right)} \leq C(R) \varepsilon\|f\|_{L^{2}(\Omega)} .
\end{gathered}
$$

Proposition 4.2. For any $R>0$, there exist two strictly positive constants $C_{1}(R)$ and $C_{2}(R)$ such that:

$$
\begin{gathered}
\left\|\widetilde{u}^{\varepsilon}-u^{0}\right\|_{H^{1}\left(\Omega_{R}\right)} \geqslant C_{1}(R) \varepsilon \sqrt{|\log (\varepsilon)|}\left|u^{0}(0,0)\right|-C_{2}(R) \varepsilon^{2}|\log (\varepsilon)|^{3 / 2}\|f\|_{L^{2}(\Omega)}, \\
\left\|\widetilde{u}^{\varepsilon}-u^{0}\right\|_{L^{2}\left(\Omega_{R}\right)} \geqslant C_{1}(R) \varepsilon\left|u^{0}(0,0)\right|-C_{2}(R) \varepsilon^{2}|\log (\varepsilon)|\|f\|_{L^{2}(\Omega)} .
\end{gathered}
$$

Reformulation of problem (2.42) in $\Omega$. As in Section 4.2, we first characterize the restriction of $\widetilde{u}^{\varepsilon}$ to $\Omega$. For this we eliminate $U^{\varepsilon}$ in (2.42). Since $U^{\varepsilon}$ is given by (2.43), we have in particular:

$$
\frac{\mathrm{d} U^{\varepsilon}}{\mathrm{d} x}(0)-i \omega U^{\varepsilon}(0)=0
$$

Using the coupling conditions between $\widetilde{u}^{\varepsilon}$ and $U^{\varepsilon}$ (last line of (2.42)), we see that $\widetilde{u}^{\varepsilon}$ satisfies on $\Sigma_{\varepsilon}$ the boundary condition:

$$
\frac{\partial \widetilde{u}^{\varepsilon}}{\partial x}-\frac{i \omega}{\varepsilon} \int_{\Sigma_{\varepsilon}} u^{\varepsilon} \mathrm{d} \sigma=0
$$


In other words, the restriction of $\widetilde{u}^{\varepsilon}$ to $\Omega$ is characterized as the unique solution of the boundary value problem:

$$
\begin{cases}-\Delta \widetilde{u}^{\varepsilon}-\omega^{2} \widetilde{u}^{\varepsilon}=f, & \text { in } \Omega, \\ \frac{\partial \widetilde{u}^{\varepsilon}}{\partial x}=0 & \text { on } \partial \Omega \backslash \Sigma_{\varepsilon}, \\ \left.\frac{\partial \widetilde{u}^{\varepsilon}}{\partial x}\right|_{\Sigma_{\varepsilon}}+T_{0}^{\varepsilon}\left(\left.\widetilde{u}^{\varepsilon}\right|_{\Sigma_{\varepsilon}}\right)=0, & \text { on } \Sigma_{\varepsilon} \\ \widetilde{u}^{\varepsilon} \text { is outgoing at infinity, } & \text { (see Rem. 2.3) }\end{cases}
$$

where the operator $T_{0}^{\varepsilon} \in \mathcal{L}\left(H^{\frac{1}{2}}\left(\Sigma_{\varepsilon}\right),\left[H^{\frac{1}{2}}\left(\Sigma_{\varepsilon}\right)\right]^{\prime}\right)$ is defined by:

$$
\left(T_{0}^{\varepsilon} \varphi\right)(y)=-\frac{i \omega}{\varepsilon} \int_{0}^{\varepsilon} \varphi(y) \mathrm{d} y \Longleftrightarrow T_{0}^{\varepsilon} \varphi=-i \omega \varphi_{0}^{\varepsilon} w_{0}^{\varepsilon}, \quad\left(\varphi_{0}^{\varepsilon} \text { given by }(4.2)\right) \text {. }
$$

Consequently, we can characterize the restriction of $\widetilde{u}^{\varepsilon}$ to $\Omega_{R}$ as the unique solution of the variational problem:

$$
\left\{\begin{array}{l}
\text { Find } \widetilde{u}^{\varepsilon} \in H^{1}\left(\Omega_{R}\right) \text { such that: } \\
a\left(\widetilde{u}^{\varepsilon}, v\right)+b_{R}\left(\widetilde{u}^{\varepsilon}, v\right)+b_{0}^{\varepsilon}\left(\widetilde{u}^{\varepsilon}, v\right)=\int_{\Omega_{R}} f \bar{v} \mathrm{~d} \mathbf{x}, \quad \forall v \in H^{1}\left(\Omega_{R}\right)
\end{array}\right.
$$

or equivalently, in the operator form:

$$
\text { Find } \widetilde{u}^{\varepsilon} \in H^{1}\left(\Omega_{R}\right) \text { such that } \quad\left(A_{R}+B_{0}^{\varepsilon}\right) \widetilde{u}^{\varepsilon}=\varphi_{f} \text {. }
$$

Remark 4.5. Comparing (4.82) and (4.45), one sees that the approximation analysis amounts, in some sense, to evaluate how the operator $B_{0}^{\varepsilon}$ is a good approximation to $B^{\varepsilon}$.

In what follows, we shall work with the equation satisfied by the "error" $u^{0}-\widetilde{u}^{\varepsilon}$, namely:

$$
\left(A_{R}+B_{0}^{\varepsilon}\right)\left(u^{0}-\widetilde{u}^{\varepsilon}\right)=B_{0}^{\varepsilon} u^{0}
$$

Stability result. We only state the result whose proof is a trivial consequence of the fact that $B_{0}^{\varepsilon}$ tends to 0 in $\mathcal{L}\left(H^{1}\left(\Omega_{R}\right)\right)$ (cf. Rem. 4.2).

Lemma 4.6. There exists a positive constant $C$ such that:

$$
\left\|\left(A_{R}+B_{0}^{\varepsilon}\right)^{-1}\right\|_{\mathcal{L}\left(H^{1}\left(\Omega_{R}\right)\right)} \leq C
$$

$H^{1}\left(\Omega_{R}\right)$-estimate of $\widetilde{u}^{\varepsilon}-u_{0}$. We show the estimate (4.73). From (4.83) and Lemma 4.6, we deduce that:

$$
\left\|\widetilde{u}^{\varepsilon}-u^{0}\right\|_{H^{1}\left(\Omega_{R}\right)} \leq C\left\|B_{0}^{\varepsilon} u^{0}\right\|_{H^{1}\left(\Omega_{R}\right)} .
$$

One concludes thanks to (4.59), (cf. Lem. 4.5). 
$H^{1+s}\left(\Omega_{R}\right)$-estimate of $\widetilde{u}^{\varepsilon}-u_{0}$. We show the estimate (4.72). The reinterpretation of equation (4.83) as a boundary value problem in $\Omega$ leads to (we set $\widetilde{e}^{\varepsilon}=\widetilde{u}^{\varepsilon}-u^{0}$ ):

$$
\begin{cases}\Delta \widetilde{e}^{\varepsilon}+\omega^{2} \widetilde{e}^{\varepsilon}=0, & \text { in } \Omega, \\ \frac{\partial \widetilde{e}^{\varepsilon}}{\partial n}=i \omega\left(\widetilde{u}^{\varepsilon} ; w_{0}^{\varepsilon}\right)_{\Sigma_{\varepsilon}} \tilde{w}_{0}^{\varepsilon}, & \text { on } \Sigma^{R},\end{cases}
$$

where $\widetilde{e}^{\varepsilon}$ satisfies moreover the outgoing radiation condition (2.11). As $\tilde{w}_{0}^{\varepsilon}$, the extension of $w_{0}^{\varepsilon}$ by zero, belongs to $H^{-\frac{1}{2}+s}(\partial \Omega)$ for any $\left.s \in\right] 0,1$ [, by localization and standard elliptic regularity estimate for Neumann problems, we obtain:

$$
\left\|\widetilde{e}^{\varepsilon}\right\|_{H^{1+s}\left(\Omega_{R}\right)} \leqslant C(s)\left|\left(\widetilde{u}^{\varepsilon} ; w_{0}^{\varepsilon}\right)_{\Sigma_{\varepsilon}}\right|\left\|\tilde{w}_{0}^{\varepsilon}\right\|_{H^{-\frac{1}{2}+s}(\mathbb{R})} .
$$

To estimate the term $\left(\widetilde{u}^{\varepsilon} ; w_{0}^{\varepsilon}\right)_{\Sigma_{\varepsilon}}$, we use the triangular:

$$
\left|\left(\widetilde{u}^{\varepsilon} ; w_{0}^{\varepsilon}\right)_{\Sigma_{\varepsilon}}\right| \leqslant\left|\left(u^{0} ; w_{0}^{\varepsilon}\right)_{\Sigma_{\varepsilon}}\right|+\left|\left(\widetilde{e}^{\varepsilon} ; w_{0}^{\varepsilon}\right)_{\Sigma_{\varepsilon}}\right| .
$$

For the first term in the right hand side we apply (4.11) (cf. Lem. 4.1) to $u=u^{0}$ and $s=1$ (we simply use the $H^{2}$ regularity result for $u^{0}$ ) while, for the second term, we use (4.73) and inequality (4.10) of Lemma 4.1. We get:

$$
\left|\left(\widetilde{u}^{\varepsilon} ; w_{0}^{\varepsilon}\right)_{\Sigma_{\varepsilon}}\right| \leqslant C \sqrt{\varepsilon}\|f\|_{L^{2}(\Omega)}+C \varepsilon^{\frac{3}{2}}|\log (\varepsilon)|\|f\|_{L^{2}(\Omega)} \leq C \sqrt{\varepsilon}\|f\|_{L^{2}(\Omega)} .
$$

One easily concludes by combining (4.87), (4.89) and inequality (4.14).

Remark 4.6. One deduces in particular the $H^{1+s}$ stability estimate:

$$
\forall s \in\left[0,1\left[, \quad\left\|\widetilde{u}^{\varepsilon}\right\|_{H^{1+s}\left(\Omega_{R}\right)} \leq C(s)\|f\|_{L^{2}(\Omega)} .\right.\right.
$$

If suffices to write $\widetilde{u}^{\varepsilon}=u^{0}+\widetilde{u}^{\varepsilon}-u^{0}$ and to use the triangular inequality to obtain:

$$
\left\|\widetilde{u}^{\varepsilon}\right\|_{H^{1+s}\left(\Omega_{R}\right)} \leq\left\|u^{0}\right\|_{H^{1+s}\left(\Omega_{R}\right)}+\left\|\widetilde{u}^{\varepsilon}-u^{0}\right\|_{H^{1+s}\left(\Omega_{R}\right)} \leq C(s)\left(1+\varepsilon^{1-s}\right)\|f\|_{L^{2}(\Omega)} .
$$

Remark 4.7. Note that the two estimates (4.72) and (4.73) (and consequently the estimate (4.90) in Rem. 4.6) remain valid even if the support of $f$ contains the origin. Indeed, their proof does not require more than the $H^{2}$ regularity for $u^{0}$.

$L^{2}\left(\Omega_{R}\right)$-estimate of $\widetilde{u}^{\varepsilon}-u_{0}$. We show the estimate (4.74). We use a standard duality technique. Let us introduce $\psi^{\varepsilon}$ solution of (let us recall that $\widetilde{e}^{\varepsilon}=\widetilde{u}^{\varepsilon}-u^{0}$ ):

$$
\mid \begin{aligned}
& \text { Find } \psi^{\varepsilon} \in H^{1}\left(\Omega_{R}\right) \text { such that } \\
& \left(A_{R} v, \psi^{\varepsilon}\right)_{H^{1}\left(\Omega_{R}\right)}=\left(v, \widetilde{e}^{\varepsilon}\right)_{L^{2}\left(\Omega_{R}\right)}, \quad \forall v \in H^{1}\left(\Omega_{R}\right) .
\end{aligned}
$$

The function $\psi^{\varepsilon}$ is also characterized as the solution of the boundary value problem:

$$
\begin{cases}\Delta \psi^{\varepsilon}+\omega^{2} \psi^{\varepsilon}=-\widetilde{e}^{\varepsilon}, & \text { in } \Omega_{R}, \\ \frac{\partial \psi^{\varepsilon}}{\partial n}=0, & \text { on } \Sigma^{R}, \\ \frac{\partial \psi^{\varepsilon}}{\partial n}+T_{R}^{*} \psi^{\varepsilon}=0, & \text { on } \Gamma_{R}\end{cases}
$$

where the operator $T_{R}^{*}$, the adjoint of $T_{R}$, is defined as $T_{R}$ (see (2.14)) by simply replacing $\mu_{n}^{R}(\omega)$ by $\overline{\mu_{n}^{R}(\omega)}$. Note that, by elliptic regularity, $\psi^{\varepsilon} \in H^{2}\left(\Omega_{R}\right)$ and:

$$
\left\|\psi^{\varepsilon}\right\|_{H^{2}\left(\Omega_{R}\right)} \leq C\left\|\widetilde{e}^{\varepsilon}\right\|_{L^{2}\left(\Omega_{R}\right)} .
$$


Let us take $v=\widetilde{e}^{\varepsilon}$ in (4.91), we get:

$$
\left(A_{R} \widetilde{e}^{\varepsilon}, \psi^{\varepsilon}\right)_{H^{1}\left(\Omega_{R}\right)}=\left\|\widetilde{e}^{\varepsilon}\right\|_{L^{2}\left(\Omega_{R}\right)}^{2} .
$$

From equations (4.46) and (4.82), we also deduce that:

$$
A_{R} \widetilde{e}^{\varepsilon}=-B_{0}^{\varepsilon} \widetilde{u}^{\varepsilon}
$$

Therefore, by definition of $B_{0}^{\varepsilon}$ :

$$
\left\|\widetilde{e}^{\varepsilon}\right\|_{L^{2}\left(\Omega_{R}\right)}^{2}=\left(A_{R} \widetilde{e}^{\varepsilon}, \psi^{\varepsilon}\right)_{H^{1}\left(\Omega_{R}\right)}=-b_{0}^{\varepsilon}\left(\widetilde{u}^{\varepsilon}, \psi^{\varepsilon}\right)=i \omega\left(\widetilde{u}^{\varepsilon}\right)_{0}^{\varepsilon}\left(\psi^{\varepsilon}\right)_{0}^{\varepsilon} .
$$

We use (4.89) (see previous paragraph) to bound $\left(\widetilde{u}^{\varepsilon}\right)_{0}^{\varepsilon}=\left(\widetilde{u}^{\varepsilon} ; w_{0}^{\varepsilon}\right)_{\Sigma_{\varepsilon}}$ and apply (4.11) to $u=\psi^{\varepsilon}$ (with $s=2$ ) to obtain, thanks to $(4.93)$ :

$$
\left\|\widetilde{e}^{\varepsilon}\right\|_{L^{2}\left(\Omega_{R}\right)}^{2} \leq C \varepsilon\|f\|_{L^{2}(\Omega)}\left\|\psi^{\varepsilon}\right\|_{H^{2}\left(\Omega_{R}\right)} \leq C \varepsilon\|f\|_{L^{2}(\Omega)}\left\|\widetilde{e}^{\varepsilon}\right\|_{L^{2}\left(\Omega_{R}\right)} .
$$

This completes the proof of Proposition 4.1.

A lower bound for $\widetilde{u}^{\varepsilon}-u_{0}$ in $H^{1}\left(\Omega_{R}\right)$. We prove (4.75). We shall use the following trace theorem (see [19], Thm. 1.5.2, p. 25):

$$
\forall u \in H^{1}\left(\Omega_{R}\right), \quad\left\|\frac{\partial u}{\partial n}\right\|_{\left[H_{00}^{1 / 2}\left(\Sigma^{R}\right)\right]^{\prime}} \leq C\left(\|u\|_{H^{1}\left(\Omega_{R}\right)}+\|\Delta u\|_{L^{2}\left(\Omega_{R}\right)}\right) .
$$

Applying (4.98) to $\widetilde{e}^{\varepsilon}$, we thus get, since $\Delta \widetilde{e}^{\varepsilon}+\omega^{2} \widetilde{e}^{\varepsilon}=0$ in $\Omega_{R}$ :

$$
\left\|\frac{\partial \widetilde{e}^{\varepsilon}}{\partial n}\right\|_{\left[H_{00}^{1 / 2}\left(\Sigma^{R}\right)\right]^{\prime}} \leq C\left\|\widetilde{e}^{\varepsilon}\right\|_{H^{1}\left(\Omega_{R}\right)},
$$

and taking into account the boundary condition satisfied by $\widetilde{e}^{\varepsilon}$ on $\Sigma^{R}$ (see (4.86)):

$$
\omega\left|\left(\widetilde{u}^{\varepsilon} ; w_{0}^{\varepsilon}\right)_{\Sigma_{\varepsilon}}\right|\left\|\tilde{w}_{0}^{\varepsilon}\right\|_{\left[H_{00}^{1 / 2}\left(\Sigma^{R}\right)\right]^{\prime}} \leq C\left\|\widetilde{e}^{\varepsilon}\right\|_{H^{1}\left(\Omega_{R}\right)} .
$$

Moreover, since $\tilde{w}_{0}^{\varepsilon}$ vanishes outside $\Sigma_{\varepsilon}$, it is easy to show (see [24]) that:

$$
\left\|\tilde{w}_{0}^{\varepsilon}\right\|_{\left[H_{00}^{1 / 2}\left(\Sigma^{R}\right)\right]^{\prime}} \geq C\left\|\tilde{w}_{0}^{\varepsilon}\right\|_{H^{-1 / 2}(\mathbb{R})}
$$

where $C$ does not depend on $\varepsilon$. Finally, thanks to (4.15) ( $c f$. Lem. 4.2) we get:

$$
\left\|\widetilde{e}^{\varepsilon}\right\|_{H^{1}\left(\Omega_{R}\right)} \geq C \sqrt{\varepsilon|\log \varepsilon|}\left|\left(\widetilde{u}^{\varepsilon} ; w_{0}^{\varepsilon}\right)_{\Sigma_{\varepsilon}}\right| .
$$

It remains to get a lower bound for $\left|\left(\widetilde{u}^{\varepsilon} ; w_{0}^{\varepsilon}\right)_{\Sigma_{\varepsilon}}\right|$. We start from:

$$
\left|\left(\widetilde{u}^{\varepsilon} ; w_{0}^{\varepsilon}\right)_{\Sigma_{\varepsilon}}\right| \geqslant\left|\left(u^{0} ; w_{0}^{\varepsilon}\right)_{\Sigma_{\varepsilon}}\right|-\left|\left(\widetilde{e}^{\varepsilon} ; w_{0}^{\varepsilon}\right)_{\Sigma_{\varepsilon}}\right| .
$$

The function $u^{0}$ is smooth at the neighborhood of $\Sigma_{\varepsilon}$, due to the fact that $f$ is compactly supported in $\Omega$. In particular, it is Lipschitz continuous in a neighborhood of the origin, the Lipschitz constant being estimated by the $L^{2}(\Omega)$-norm of $f$. More precisely, we can write locally:

$$
\left|u^{0}(0, y)-u^{0}(0,0)\right| \leqslant C y\|f\|_{L^{2}(\Omega)},
$$


where the constant $C$ only depends of the neighborhood and the distance from the support of $f$ to the origin (this follows from elliptic regularity). From (4.103), we deduce:

$$
\left|\left(u^{0} ; w_{0}^{\varepsilon}\right)_{\Sigma_{\varepsilon}}\right| \geq C \sqrt{\varepsilon}\left|u^{0}(0,0)\right|-C \varepsilon^{3 / 2}\|f\|_{L^{2}(\Omega)} .
$$

On the other hand, applying successively (4.10) to $\widetilde{e}^{\varepsilon}$ and estimate (4.73) of Proposition 4.1, we get the estimate:

$$
\left|\left(\widetilde{e}^{\varepsilon} ; w_{0}^{\varepsilon}\right)_{\Sigma_{\varepsilon}}\right| \leq C \varepsilon \sqrt{|\log \varepsilon|}\left\|\widetilde{e}^{\varepsilon}\right\|_{H^{1}\left(\Omega_{R}\right)} \leq C \varepsilon^{3 / 2}|\log \varepsilon|\|f\|_{L^{2}(\Omega)} .
$$

One concludes from (4.101), (4.102), (4.104) and (4.105).

A lower bound for $\widetilde{u}^{\varepsilon}-u_{0}$ in $L^{2}\left(\Omega_{R, R^{\prime}}\right)$. We give here a "direct" proof of (4.76) that makes use of an integral representation of $\widetilde{e}^{\varepsilon}$ (more indirect proofs are also possible). Since $\widetilde{e}^{\varepsilon}$ is the outgoing solution of the non homogeneous Neumann half-space problem (4.86), it admits the following integral representation:

$$
\left\{\begin{array}{l}
\widetilde{e}^{\varepsilon}(x, y)=-i \omega \frac{\left(\widetilde{u}^{\varepsilon} ; w_{0}^{\varepsilon}\right) \Sigma_{\varepsilon}}{\sqrt{\varepsilon}} \int_{0}^{\varepsilon} G\left(x, y, y^{\prime}\right) \mathrm{d} y^{\prime} \\
G\left(x, y, y^{\prime}\right)=\frac{1}{2 i} H_{0}^{(1)}\left(\omega \sqrt{x^{2}+\left(y-y^{\prime}\right)^{2}}\right) .
\end{array}\right.
$$

Using polar coordinates $(x=r \sin \theta, y=r \cos \theta)$, we can write:

$$
G\left(x, y, y^{\prime}\right)=\frac{1}{2 i} H_{0}^{(1)}\left(\omega r\left[1-2 \frac{y^{\prime}}{r} \cos \theta+\left(\frac{y^{\prime}}{r}\right)^{2}\right]^{\frac{1}{2}}\right)
$$

Since the Hankel function $H_{0}^{(1)}$ is analytic except at the origin, for any $0<R^{\prime}<R$ we deduce the existence of a constant $C$, depending only on $R, R^{\prime}$ and $\omega$ such that:

$$
\left.\forall(x, y) \in \Omega_{R, R^{\prime}}, \quad \forall y^{\prime} \in\right] 0, \varepsilon\left[, \quad\left|G\left(x, y, y^{\prime}\right)-\frac{1}{2 i} H_{0}^{(1)}(\omega r)\right| \leq C \varepsilon .\right.
$$

As a consequence, we have:

$$
\forall(x, y) \in \Omega_{R, R^{\prime}}, \quad\left|\widetilde{e}^{\varepsilon}(x, y)+\frac{\omega}{2} \sqrt{\varepsilon}\left(\widetilde{u}^{\varepsilon} ; w_{0}^{\varepsilon}\right)_{\Sigma_{\varepsilon}} H_{0}^{(1)}(\omega r)\right| \leq C \varepsilon^{\frac{3}{2}}\left|\left(\widetilde{u}^{\varepsilon} ; w_{0}^{\varepsilon}\right)_{\Sigma_{\varepsilon}}\right|,
$$

from which we deduce in particular that:

$$
\forall(x, y) \in \Omega_{R, R^{\prime}}, \quad\left|\widetilde{e}^{\varepsilon}(x, y)\right|^{2} \geq \frac{\omega^{2}}{4} \varepsilon\left(\widetilde{u}^{\varepsilon} ; w_{0}^{\varepsilon}\right)_{\Sigma_{\varepsilon}}^{2}\left\{\left|H_{0}^{(1)}(\omega r)\right|^{2}-C \varepsilon\right\} .
$$

We integrate (4.110) over $\Omega_{R, R^{\prime}}$, take the square root and get, with $C_{1}>0$ :

$$
\left\|\widetilde{e}^{\varepsilon}\right\|_{L^{2}\left(\Omega_{R, R^{\prime}}\right)}^{2} \geq\left(C_{1} \varepsilon^{\frac{1}{2}}-C_{2} \varepsilon^{\frac{3}{2}}\right)\left|\left(\widetilde{u}^{\varepsilon} ; w_{0}^{\varepsilon}\right)_{\Sigma_{\varepsilon}}\right| .
$$

Finally, (3.9) results from (4.111) and (4.104). The proof of proposition 4.2 is complete.

\section{Proofs of theorems 3.3 and 3.4}

\subsection{Study of the error in the half-space $\Omega$}

$H^{1}\left(\Omega_{R}\right)$-estimate of $\widetilde{u}^{\varepsilon}-u^{\varepsilon}$. We prove (3.11). Let us introduce the error $\eta^{\varepsilon}$ :

$$
\eta^{\varepsilon}=\widetilde{u}^{\varepsilon}-u^{\varepsilon},
$$


generated by the approximate model. It is easy to see that $\eta^{\varepsilon}$ satisfies indifferently:

$$
\left(A_{R}+B^{\varepsilon}\right) \eta^{\varepsilon}=R^{\varepsilon} \tilde{u}^{\varepsilon} \quad \text { and } \quad\left(A_{R}+B_{0}^{\varepsilon}\right) \eta^{\varepsilon}=R^{\varepsilon} u^{\varepsilon}
$$

By Lemma 4.4, we deduce:

$$
\left\|\eta^{\varepsilon}\right\|_{H^{1}\left(\Omega_{R}\right)} \leqslant C\left\|R^{\varepsilon} \widetilde{u}^{\varepsilon}\right\|_{H^{1}\left(\Omega_{R}\right)} \leqslant C\left\|R^{\varepsilon} u^{0}\right\|_{H^{1}\left(\Omega_{R}\right)}+\left\|R^{\varepsilon}\left(\widetilde{u}^{\varepsilon}-u^{0}\right)\right\|_{H^{1}\left(\Omega_{R}\right)} .
$$

We can estimate $R^{\varepsilon} u^{0}$ thanks to (4.60) (Lem. 4.5). For the second term, using inequality (4.66) (see Proof of Lem. 4.5), we can write:

$$
\left\|R^{\varepsilon}\left(\widetilde{u}^{\varepsilon}-u^{0}\right)\right\|_{H^{1}\left(\Omega_{R}\right)} \leq C\left\|\widetilde{u}^{\varepsilon}-u^{0}\right\|_{H_{*}^{\frac{1}{2}}\left(\Sigma^{\varepsilon}\right)} .
$$

Applying (4.22) (cf. Lem. 4.3) to $u=\widetilde{u}^{\varepsilon}-u^{0}$ with $s=1 / 2$, we get:

$$
\left\|\widetilde{u}^{\varepsilon}-u^{0}\right\|_{H_{*}^{\frac{1}{2}}\left(\Sigma^{\varepsilon}\right)} \leqslant C \sqrt{\varepsilon}\left\|\widetilde{u}^{\varepsilon}-u^{0}\right\|_{H^{\frac{3}{2}}(\Omega)} .
$$

Then, thanks to estimate (4.72) of Proposition 4.1 (we take $s=1 / 2$ ), we have:

$$
\left\|\widetilde{u}^{\varepsilon}-u^{0}\right\|_{H_{*}^{\frac{1}{2}}\left(\Sigma^{\varepsilon}\right)} \leqslant C \varepsilon\|f\|_{L^{2}(\Omega)} .
$$

One concludes easily.

Remark 5.1. An (quasi) obvious consequence of (3.11) is that:

$$
\left\|u^{\varepsilon}\right\|_{H_{*}^{\frac{1}{2}}\left(\Sigma^{\varepsilon}\right)} \leq C \varepsilon\|f\|_{L^{2}(\Omega)}
$$

Indeed by triangular inequality and trace estimate (4.22), we have:

$$
\left\{\begin{aligned}
\left\|u^{\varepsilon}\right\|_{H_{*}^{\frac{1}{2}}\left(\Sigma^{\varepsilon}\right)} & \leq\left\|u^{0}\right\|_{H_{*}^{\frac{1}{2}}\left(\Sigma^{\varepsilon}\right)}+\left\|\widetilde{u}^{\varepsilon}-u^{0}\right\|_{H_{*}^{\frac{1}{2}}\left(\Sigma^{\varepsilon}\right)}+\left\|u^{\varepsilon}-\widetilde{u}^{\varepsilon}\right\|_{H_{*}^{\frac{1}{2}}\left(\Sigma^{\varepsilon}\right)} \\
& \leq\left\|u^{0}\right\|_{H_{*}^{\frac{1}{2}}\left(\Sigma^{\varepsilon}\right)}+C \sqrt{\varepsilon}\left\|\widetilde{u}^{\varepsilon}-u^{0}\right\|_{H^{3 / 2}\left(\Omega_{R}\right)}+C\left\|u^{\varepsilon}-\widetilde{u}^{\varepsilon}\right\|_{H^{1}\left(\Omega_{R}\right)}
\end{aligned}\right.
$$

As the support of $f$ does not contain the origin we can apply (4.68) - see the proof of Lemma 4.5, namely:

$$
\left\|u^{0}\right\|_{H_{*}^{\frac{1}{2}}\left(\Sigma^{\varepsilon}\right)} \leq C \varepsilon\|f\|_{L^{2}(\Omega)}
$$

Therefore one deduces (5.7) from (3.11) and (4.72). Note that (5.7) requires that the support of $f$ does not contain the origin.

$L^{2}\left(\Omega_{R}\right)$-estimate of $\widetilde{u}^{\varepsilon}-u^{\varepsilon}$. We prove $(3.12)$ by a duality technique. Let us consider the problem: Let us introduce $\widetilde{\Psi}^{\varepsilon}$ solution of (let us recall that $\eta^{\varepsilon}=\widetilde{u}^{\varepsilon}-u^{\varepsilon}$ ):

$$
\mid \begin{aligned}
& \text { Find } \widetilde{\Psi}^{\varepsilon} \in H^{1}\left(\Omega_{R}\right) \text { such that } \\
& \left(\left(A_{R}+B_{0}^{\varepsilon}\right) v, \widetilde{\Psi}^{\varepsilon}\right)_{H^{1}\left(\Omega_{R}\right)}=\left(v, \eta^{\varepsilon}\right)_{L^{2}\left(\Omega_{R}\right)}, \quad \forall v \in H^{1}\left(\Omega_{R}\right) .
\end{aligned}
$$


The function $\widetilde{\Psi}^{\varepsilon}$ is also characterized as the solution on the boundary value problem:

$$
\begin{cases}\Delta \widetilde{\Psi}^{\varepsilon}+\omega^{2} \widetilde{\Psi}^{\varepsilon}=-\eta^{\varepsilon}, & \text { in } \Omega_{R}, \\ \frac{\partial \widetilde{\Psi}^{\varepsilon}}{\partial n}+i \omega\left(\widetilde{\Psi}^{\varepsilon}, w_{0}^{\varepsilon}\right)_{\Sigma_{\varepsilon}} \widetilde{w}_{0}^{\varepsilon}=0, & \text { on } \Sigma^{R}, \\ \frac{\partial \widetilde{\Psi}^{\varepsilon}}{\partial n}+T_{R}^{*} \widetilde{\Psi}^{\varepsilon}=0, & \text { on } \Gamma_{R} .\end{cases}
$$

Up to the change of $T_{R}$ into $T_{R}^{*}$ and $T_{0}^{\varepsilon}$ into its adjoint $\left(T_{0}^{\varepsilon}\right)^{*}$ (simply change $i \omega$ into $-i \omega$ in definition (4.80)), the relationship $\eta^{\varepsilon} \mapsto \widetilde{\Psi}^{\varepsilon}$ is the same as the relationship $f \mapsto \widetilde{u}^{\varepsilon}$. We can thus apply the equivalent of the stability estimate (4.90) (see Rems. 4.6 and 4.7), which leads to:

$$
\forall s \in\left[0,1\left[, \quad \widetilde{\Psi}^{\varepsilon} \in H^{1+s}(\Omega), \quad\left\|\widetilde{\Psi}^{\varepsilon}\right\|_{H^{1+s}(\Omega)} \leqslant C(s)\left\|\eta^{\varepsilon}\right\|_{L^{2}(\Omega)} .\right.\right.
$$

Let us take $v=\eta^{\varepsilon}$ in (5.10), from (5.2), we get:

$$
\left\|\eta^{\varepsilon}\right\|_{L^{2}\left(\Omega_{R}\right)}^{2}=\left(\left(A+B^{\varepsilon, 0}\right) \eta^{\varepsilon}, \widetilde{\Psi}^{\varepsilon}\right)=\left(R^{\varepsilon} u^{\varepsilon}, \widetilde{\Psi}^{\varepsilon}\right)=r^{\varepsilon}\left(u^{\varepsilon}, \widetilde{\Psi}^{\varepsilon}\right) .
$$

Therefore, applying inequality (4.65) for $r^{\varepsilon}(.,$.$) :$

$$
\left\|\eta^{\varepsilon}\right\|_{L^{2}\left(\Omega_{R}\right)}^{2} \leq C\left\|u^{\varepsilon}\right\|_{H_{*}^{\frac{1}{2}}\left(\Sigma_{\varepsilon}\right)} \cdot\left\|\widetilde{\Psi}^{\varepsilon}\right\|_{H_{*}^{\frac{1}{2}}\left(\Sigma_{\varepsilon}\right)} .
$$

On the other hand, by (4.22) (Lem. 4.3) and (5.12), we have:

$$
\forall s \in\left[0,1\left[, \quad\left\|\widetilde{\Psi}^{\varepsilon}\right\|_{H_{*}^{\frac{1}{2}}\left(\Sigma^{\varepsilon}\right)} \leqslant C(s) \varepsilon^{s}\left\|\widetilde{\Psi}^{\varepsilon}\right\|_{H^{1+s}(\Omega)} \leqslant C(s) \varepsilon^{s}\left\|\eta^{\varepsilon}\right\|_{L^{2}(\Omega)} .\right.\right.
$$

One deduces (3.12) from (5.7), (5.14) and (5.15).

Estimates of $\widetilde{u}^{\varepsilon}-u^{\varepsilon}$ in the domains $\Omega_{R, R^{\prime}}$. We first prove an $L^{2}$ estimate. Let us introduce $\widetilde{\Psi}_{R^{\prime}}^{\varepsilon}$ the solution of problem (5.10) (or (5.11) ) in which we have replaced $\eta^{\varepsilon}$ by:

$$
\eta_{R^{\prime}}^{\varepsilon}=\left.\eta^{\varepsilon}\right|_{\Omega_{R, R^{\prime}}}
$$

Since $\left(\left(A_{R}+B_{0}^{\varepsilon}\right) v, \widetilde{\Psi}_{R^{\prime}}^{\varepsilon}\right)_{H^{1}\left(\Omega_{R}\right)}=\left(v, \eta_{R^{\prime}}^{\varepsilon}\right)_{L^{2}\left(\Omega_{R}\right)}$ for any $v \in H^{1}\left(\Omega_{R}\right)$, we have in particular, with $v=\eta^{\varepsilon}$ :

$$
\left\|\eta^{\varepsilon}\right\|_{L^{2}\left(\Omega_{R, R^{\prime}}\right)}^{2}=\left(\eta^{\varepsilon}, \eta_{R^{\prime}}^{\varepsilon}\right)_{L^{2}\left(\Omega_{R, R^{\prime}}\right)}=\left(\left(A_{R}+B_{0}^{\varepsilon}\right) \eta^{\varepsilon}, \widetilde{\Psi}_{R^{\prime}}^{\varepsilon}\right)_{H^{1}\left(\Omega_{R}\right)}=r^{\varepsilon}\left(u^{\varepsilon}, \widetilde{\Psi}_{R^{\prime}}^{\varepsilon}\right) .
$$

Thus, applying (4.65) once again:

$$
\left\|\eta^{\varepsilon}\right\|_{L^{2}\left(\Omega_{R, R^{\prime}}\right)}^{2} \leq C\left\|u^{\varepsilon}\right\|_{H_{*}^{\frac{1}{2}}\left(\Sigma_{\varepsilon}\right)} \cdot\left\|\widetilde{\Psi}_{R^{\prime}}^{\varepsilon}\right\|_{H_{*}^{\frac{1}{2}}\left(\Sigma_{\varepsilon}\right)} \cdot
$$

The novelty with respect of the previous proof is due to the fact that, since $\eta_{R^{\prime}}^{\varepsilon}$ is supported in $\Omega_{R, R^{\prime}}$, we can apply the estimate (5.7) (see rem. 5.1), replacing $\widetilde{u}^{\varepsilon}$ by $\widetilde{\Psi}_{R^{\prime}}^{\varepsilon}$ and $f$ by $\eta_{R^{\prime}}^{\varepsilon}$ :

$$
\left\|\widetilde{\Psi}_{R^{\prime}}^{\varepsilon}\right\|_{H_{*}^{\frac{1}{2}}\left(\Sigma_{\varepsilon}\right)} \leq C\left(R^{\prime}\right) \varepsilon\left\|\eta^{\varepsilon}\right\|_{L^{2}\left(\Omega_{R, R^{\prime}}\right)} .
$$

We thus get $\left\|\eta^{\varepsilon}\right\|_{L^{2}\left(\Omega_{R, R^{\prime}}\right)} \leq C \varepsilon\left\|u^{\varepsilon}\right\|_{H_{*}^{\frac{1}{2}}\left(\Sigma_{\varepsilon}\right)}$, which, thanks to (5.7), leads to

$$
\left\|\eta^{\varepsilon}\right\|_{L^{2}\left(\Omega_{R, R^{\prime}}\right)} \leq C \varepsilon^{2}\|f\|_{L^{2}(\Omega)} .
$$


It is now easy to derive the local $H^{1}$ estimate (3.13) by localization. Thanks to (5.19), we only have to estimate the $L^{2}$ norm of $\nabla \eta^{\varepsilon}$. Let $R_{1}<R^{\prime}<R<R_{2}$ and $\theta$ be a real valued cut-off function in $C_{0}^{\infty}\left(\mathbb{R}^{2}\right)$ such that $0 \leq \theta(x) \leq 1$ and:

$$
\operatorname{supp} \theta \subset \Omega_{R_{1}, R_{2}}, \quad \theta(x)=1 \text { in } \Omega_{R, R^{\prime}} .
$$

Since $\Delta \eta^{\varepsilon}=-\omega^{2} \eta^{\varepsilon}$, we have, in the sense of distributions in $\Omega$ :

$$
\Delta\left(\theta \eta^{\varepsilon}\right)=\left(\Delta \theta-\omega^{2} \theta\right) \eta^{\varepsilon}+2 \nabla \theta \cdot \nabla \eta^{\varepsilon}
$$

We multiply (5.20) by $\theta \overline{\eta^{\varepsilon}}$ and integrate over $\Omega$. We get:

$$
\int_{\Omega}\left|\nabla\left(\theta \eta^{\varepsilon}\right)\right|^{2} \mathrm{~d} \mathbf{x}=\int_{\Omega}\left(\Delta \theta-\omega^{2} \theta\right) \theta\left|\eta^{\varepsilon}\right|^{2}+2 \int_{\Omega} \nabla \theta \cdot \nabla \eta^{\varepsilon} \theta \overline{\eta^{\varepsilon}} \mathrm{d} \mathbf{x},
$$

that is to say, using $\theta \nabla \eta^{\varepsilon}=\nabla\left(\theta \eta^{\varepsilon}\right)-\eta^{\varepsilon} \nabla \theta$ :

$$
\int_{\Omega}\left|\nabla\left(\theta \eta^{\varepsilon}\right)\right|^{2} \mathrm{~d} \mathbf{x}=\int_{\Omega}\left[\left(\Delta \theta-\omega^{2} \theta\right) \theta-2|\nabla \theta|^{2}\right]\left|\eta^{\varepsilon}\right|^{2} \mathrm{~d} \mathbf{x}+2 \int_{\Omega} \nabla\left(\theta \eta^{\varepsilon}\right) \nabla \theta \overline{\eta^{\varepsilon}} \mathrm{d} \mathbf{x} .
$$

Using $2 a b \leq\left(a^{2} / 2+2 b^{2}\right)$ in the last term, we finally obtain:

$$
\frac{1}{2} \int_{\Omega}\left|\nabla\left(\theta \eta^{\varepsilon}\right)\right|^{2} \mathrm{~d} \mathbf{x} \leq \int_{\Omega}\left[\left(\Delta \theta-\omega^{2} \theta\right) \theta\right]\left|\eta^{\varepsilon}\right|^{2} \mathrm{~d} \mathbf{x}
$$

Using the properties of the function $\theta$ we can write:

$$
\left\|\nabla \eta^{\varepsilon}\right\|_{L^{2}\left(\Omega_{R, R^{\prime}}\right)}^{2} \leq \int_{\Omega}\left|\nabla\left(\theta \eta^{\varepsilon}\right)\right|^{2} \mathrm{~d} \mathbf{x} \leq C\left\|\eta^{\varepsilon}\right\|_{L^{2}\left(\Omega_{R_{1}, R_{2}}\right)}^{2} .
$$

One concludes by using (5.19) with $\left(R^{\prime}, R\right)=\left(R_{1}, R_{2}\right)$. The Proof of Theorem 3.3 is completed.

Lower bounds for $u^{\varepsilon}-u^{0}$ in $H^{1}\left(\Omega_{R}\right)$ and $L^{2}\left(\Omega_{R, R^{\prime}}\right)$. To obtain (3.8), it suffices to apply the triangular inequality:

$$
\left\|u^{\varepsilon}-u^{0}\right\|_{H^{1}\left(\Omega_{R, R^{\prime}}\right)} \geq\left\|\widetilde{u}^{\varepsilon}-u^{0}\right\|_{H^{1}\left(\Omega_{R, R^{\prime}}\right)}-\left\|u^{\varepsilon}-\widetilde{u}^{\varepsilon}\right\|_{H^{1}\left(\Omega_{R, R^{\prime}}\right)},
$$

and to use the inequalities (4.75) and (3.11). The proof of (3.9) is similar. This completes the Proof of Theorem 3.2.

\subsection{Study of the error in the $\operatorname{slot} \mathcal{O}_{\varepsilon}$}

Estimates of $u^{\varepsilon}-\widetilde{u}^{\varepsilon}$ in $\mathcal{O}_{\varepsilon}$. We first prove (3.18). Let us recall the expressions of $u^{\varepsilon}$ and $\widetilde{u}^{\varepsilon}$ in $\mathcal{O}_{\varepsilon}$ :

$$
\left\{\begin{array}{l}
u^{\varepsilon}(x, y)=\left(u^{\varepsilon}, w_{0}^{\varepsilon}\right)_{\Sigma_{\varepsilon}} w_{0}^{\varepsilon}(y) \exp (i \omega x)+\sum_{n=1}^{+\infty}\left(u^{\varepsilon}, w_{n}^{\varepsilon}\right)_{\Sigma_{\varepsilon}} w_{n}^{\varepsilon}(y) \mathrm{e}^{-\xi_{n}^{\varepsilon}(\omega) x} \\
\widetilde{u}^{\varepsilon}(x, y)=\left(\widetilde{u}^{\varepsilon}, w_{0}^{\varepsilon}\right)_{\Sigma_{\varepsilon}} w_{0}^{\varepsilon}(y) \exp (i \omega x) .
\end{array}\right.
$$

It is clear that the study of the error passes by the estimate of the series appearing in the second term in the expression of the difference $\left(u^{\varepsilon}, w_{0}^{\varepsilon}\right)_{\Sigma_{\varepsilon}}-\left(\widetilde{u}^{\varepsilon}, w_{0}^{\varepsilon}\right)_{\Sigma_{\varepsilon}}$. More precisely:

$$
\left\{\begin{aligned}
\left\|u^{\varepsilon}-\widetilde{u}^{\varepsilon}\right\|_{H^{1}\left(\mathcal{O}_{\varepsilon}^{L}\right)}^{2}= & \left(1+\omega^{2}\right) L\left(u^{\varepsilon}-\widetilde{u}^{\varepsilon}, w_{0}^{\varepsilon}\right)_{\Sigma_{\varepsilon}}^{2} \\
& +\sum_{n=1}^{+\infty}\left(1+2 \xi_{n}^{\varepsilon}(\omega)^{2}\right) \frac{1-\mathrm{e}^{-2 \xi_{n}^{\varepsilon}(\omega) L}}{2 \xi_{n}^{\varepsilon}(\omega)}\left(u^{\varepsilon}, w_{n}^{\varepsilon}\right)_{\Sigma_{\varepsilon}}^{2} .
\end{aligned}\right.
$$


Thanks to $\left|\xi_{n}^{\varepsilon}(\omega)\right| \leqslant\left(1+\pi^{2} n^{2} / \varepsilon^{2}\right)^{\frac{1}{2}}$ and to the fact that the function $x \mapsto\left(1-\mathrm{e}^{-x}\right)$ is bounded in $\mathbb{R}^{+}$, this can be rewritten:

$$
\left\|u^{\varepsilon}-\widetilde{u}^{\varepsilon}\right\|_{H^{1}\left(\mathcal{O}_{\varepsilon}^{L}\right)}^{2} \leq\left(1+\omega^{2}\right) L\left(u^{\varepsilon}-\widetilde{u}^{\varepsilon}, w_{0}^{\varepsilon}\right)_{\Sigma_{\varepsilon}}^{2}+C\left\|u^{\varepsilon}\right\|_{H_{*}^{\frac{1}{2}}\left(\Sigma_{\varepsilon}\right)}^{2} .
$$

Applying (4.10) of Lemma 4.1 (to $u^{\varepsilon}-\widetilde{u}^{\varepsilon}$ ) and (3.11) we get:

$$
\left|\left(u^{\varepsilon}-\widetilde{u}^{\varepsilon}, w_{0}^{\varepsilon}\right)_{\Sigma_{\varepsilon}}\right| \leq C \sqrt{\varepsilon|\log \varepsilon|}\left\|u^{\varepsilon}-\widetilde{u}^{\varepsilon}\right\|_{H^{1}\left(\Omega_{R}\right)} \leq C \varepsilon^{\frac{3}{2}} \sqrt{|\log \varepsilon|}\|f\|_{L^{2}(\Omega)} .
$$

One obtains (3.18) thanks to (5.27), (5.28) and (5.7).

For the $L^{2}$ norm, we have the formula:

$$
\left\|u^{\varepsilon}-\widetilde{u}^{\varepsilon}\right\|_{L^{2}\left(\mathcal{O}_{\varepsilon}^{L}\right)}^{2}=L\left(u^{\varepsilon}-\widetilde{u}^{\varepsilon}, w_{0}^{\varepsilon}\right)_{\Sigma_{\varepsilon}}^{2}+\sum_{n=1}^{+\infty} \frac{1-\mathrm{e}^{-2 \xi_{n}^{\varepsilon}(\omega) L}}{2 \xi_{n}^{\varepsilon}(\omega)}\left(u^{\varepsilon}, w_{n}^{\varepsilon}\right)_{\Sigma_{\varepsilon}}^{2} .
$$

Observing that for all $n \geq 1, \xi_{n}^{\varepsilon}(\omega)^{-1} \leq \xi_{1}^{\varepsilon}(\omega)^{-1} \leq C \varepsilon$, we get the (rough) estimate:

$$
\left\|u^{\varepsilon}-\widetilde{u}^{\varepsilon}\right\|_{L^{2}\left(\mathcal{O}_{\varepsilon}^{L}\right)}^{2} \leq L\left(u^{\varepsilon}-\widetilde{u}^{\varepsilon}, w_{0}^{\varepsilon}\right)_{\Sigma_{\varepsilon}}^{2}+C \varepsilon^{2}\left\|u^{\varepsilon}\right\|_{H_{*}^{\frac{1}{2}}\left(\Sigma_{\varepsilon}\right)}^{2} .
$$

One obtains (3.19) by using once again (5.7) and (5.28).

We finally prove (3.20). In the domain $\mathcal{O}_{\varepsilon}^{L, L^{\prime}}$ we have:

$$
\left\{\begin{aligned}
\left\|u^{\varepsilon}-\widetilde{u}^{\varepsilon}\right\|_{H^{1}\left(\mathcal{O}_{\varepsilon}^{L, L^{\prime}}\right)}^{2} & =\left(1+\omega^{2}\right)\left(L-L^{\prime}\right)\left(u^{\varepsilon}-\widetilde{u}^{\varepsilon}, w_{0}^{\varepsilon}\right)_{\Sigma_{\varepsilon}}^{2} \\
& +\sum_{n=1}^{+\infty}\left(1+2 \xi_{n}^{\varepsilon}(\omega)^{2}\right)\left(\frac{\mathrm{e}^{-2 \xi_{n}^{\varepsilon}(\omega) L^{\prime}}-\mathrm{e}^{-2 \xi_{n}^{\varepsilon}(\omega) L}}{2 \xi_{n}^{\varepsilon}(\omega)}\right)\left(u^{\varepsilon}, w_{n}^{\varepsilon}\right)_{\Sigma_{\varepsilon}}^{2},
\end{aligned}\right.
$$

which yields

$$
\left\|u^{\varepsilon}-\widetilde{u}^{\varepsilon}\right\|_{H^{1}\left(\mathcal{O}_{\varepsilon}^{L, L^{\prime}}\right)}^{2} \leq\left(1+\omega^{2}\right) L\left(u^{\varepsilon}-\widetilde{u}^{\varepsilon}, w_{0}^{\varepsilon}\right)_{\Sigma_{\varepsilon}}^{2}+C \mathrm{e}^{-2 \xi_{1}^{\varepsilon}(\omega) L^{\prime}}\left\|u^{\varepsilon}\right\|_{H_{*}^{2}}^{2}\left(\Sigma_{\varepsilon}\right)
$$

The conclusion is immediate since $\mathrm{e}^{-2 \xi_{1}^{\varepsilon}(\omega) L^{\prime}} \leq \mathrm{e}^{-2 a \frac{L^{\prime}}{\varepsilon}}$ for some $a>0$. The Proof of Theorem 3.4 is complete.

A lower bound for $u^{\varepsilon}$ inside the slot. In order to be complete, in particular to justify the table 2, we need to show the double inequality (3.21). Of course, it suffices to show the upper bound for $H=H^{1}\left(\mathcal{O}_{\varepsilon}^{L}\right)$ and the lower bound for $H=L^{2}\left(\mathcal{O}_{\varepsilon}^{L, L^{\prime}}\right)$. We prove below this lower bound. The proof of the upper bound, straightforward, is left to the reader. First, we calculate:

$$
\left\|u^{\varepsilon}\right\|_{L^{2}\left(\mathcal{O}_{\varepsilon}^{L, L^{\prime}}\right)}^{2}=\left(L-L^{\prime}\right)\left(u^{\varepsilon}, w_{0}^{\varepsilon}\right)_{\Sigma_{\varepsilon}}^{2}+\sum_{n=1}^{+\infty}\left(\frac{\mathrm{e}^{-2 \xi_{n}^{\varepsilon}(\omega) L^{\prime}}-\mathrm{e}^{-2 \xi_{n}^{\varepsilon}(\omega) L}}{2 \xi_{n}^{\varepsilon}(\omega)}\right)\left(u^{\varepsilon}, w_{n}^{\varepsilon}\right)_{\Sigma_{\varepsilon}}^{2} .
$$

As all the $\xi_{n}^{\varepsilon}(\omega)$ are (uniformly in $\varepsilon$ ) bounded from below for $n \geq 1$, the series in the right hand side of (5.33) converges to 0 exponentially fast. Therefore:

$$
\left\|u^{\varepsilon}\right\|_{L^{2}\left(\mathcal{O}_{\varepsilon}^{L, L^{\prime}}\right)} \geq\left|L-L^{\prime}\right|^{\frac{1}{2}}\left(u^{\varepsilon}, w_{0}^{\varepsilon}\right)_{\Sigma_{\varepsilon}}-C \mathrm{e}^{-a \frac{L^{\prime}}{\varepsilon}}\|f\|_{L^{2}(\Omega)} .
$$

Moreover:

Reasoning as for (5.28), we get:

$$
\left|\left(u^{\varepsilon}, w_{0}^{\varepsilon}\right)_{\Sigma_{\varepsilon}}\right| \geq\left|\left(u^{0}, w_{0}^{\varepsilon}\right)_{\Sigma_{\varepsilon}}\right|-\left|\left(u^{\varepsilon}-u^{0}, w_{0}^{\varepsilon}\right)_{\Sigma_{\varepsilon}}\right| .
$$

$$
\left|\left(u^{\varepsilon}-u^{0}, w_{0}^{\varepsilon}\right)_{\Sigma_{\varepsilon}}\right| \leq C \sqrt{\varepsilon|\log \varepsilon|}\left\|u^{\varepsilon}-u^{0}\right\|_{H^{1}\left(\Omega_{R}\right)} \leq C \varepsilon^{\frac{3}{2}}|\log \varepsilon|\|f\|_{L^{2}(\Omega)},
$$


The conclusion follows from (5.34), (5.35), (5.36) and (4.104).

\section{Numerical ReSults}

For the numerical simulations, we have chosen to consider a situation which is slightly different from the one of Section 2. Here, the waves are no longer generated by a source term $f$. We study the diffraction of a plane wave of incidence angle $\theta$ (with respect to the plane $x=0$ ):

$$
u^{i}(\mathbf{x}, t)=\exp i \omega(x \cos \theta+y \sin \theta)
$$

The presence of the boundary $\partial \Omega^{\varepsilon}$ induces two phenomena:

- a reflected wave due to the boundary $\partial \Omega_{\varepsilon} \backslash \partial \mathcal{O}_{\varepsilon}$;

- a diffracted wave due to the slot (plus the propagation of a wave inside the slot).

More precisely, in the absence of a slot, the solution $u^{0}$ corresponds to a pure reflection:

$$
u^{0}(\mathbf{x}, t)=\exp i \omega(x \cos \theta+y \sin \theta)+\exp i \omega(-x \cos \theta+y \sin \theta),
$$

and the diffracted field due to the slot, namely $u^{\varepsilon}=u_{t}^{\varepsilon}-u^{0}$ is therefore the solution of the following problem:

$$
\begin{cases}-\Delta u^{\varepsilon}-\omega^{2} u^{\varepsilon}=0, & \text { in } \Omega, \\ \frac{\partial u^{\varepsilon}}{\partial n}=0, & \text { on } \partial \Omega \backslash \Sigma_{\varepsilon}, \\ \left.\frac{\partial u^{\varepsilon}}{\partial n}\right|_{\Sigma_{\varepsilon}}+T^{\varepsilon}\left(\left.u^{\varepsilon}\right|_{\Sigma_{\varepsilon}}\right)=-T^{\varepsilon}\left(\left.u^{0}\right|_{\Sigma_{\varepsilon}}\right), & \text { on } \Sigma_{\varepsilon}, \\ u^{\varepsilon} \text { is outgoing at infinity. } & \end{cases}
$$

Our method consists in approximating $u^{\varepsilon}$ by $\widetilde{u}^{\varepsilon}$ solution of the following problem:

$$
\begin{cases}-\Delta \tilde{u}^{\varepsilon}-\omega^{2} \tilde{u}^{\varepsilon}=0, & \text { in } \Omega, \\ \frac{\partial \tilde{u}^{\varepsilon}}{\partial n}=0, & \text { on } \partial \Omega \backslash \Sigma_{\varepsilon} \\ \left.\frac{\partial \tilde{u}^{\varepsilon}}{\partial n}\right|_{\Sigma_{\varepsilon}}+T_{0}^{\varepsilon}\left(\left.\tilde{u}^{\varepsilon}\right|_{\Sigma_{\varepsilon}}\right)=-T_{0}^{\varepsilon}\left(\left.u^{0}\right|_{\Sigma_{\varepsilon}}\right), & \text { on } \Sigma_{\varepsilon} \\ \tilde{u}^{\varepsilon} \text { is outgoing at infinity. } & \end{cases}
$$

To perform the numerical computation of $u^{\varepsilon}$ and $\widetilde{u}^{\varepsilon}$, we use the variational formulation of problems (6.3) and (6.4) truncated to the domain $\Omega_{R}$ (we omit the details - see Sect. 2 and [17]). We use a (large order) truncation of the series appearing in the definition of the bilinear forms $b_{R}(.,$.$) and b_{\varepsilon}(.,$.$) . We apply a P_{2}$ finite element method for the space discretization. The computational code we used is the code MELINA [30] on a very thin mesh.

We first present numerical results corresponding to the following set of data:

- the pulsation $\omega$ is $\omega=2 \pi$ which corresponds to a wavelength: $\lambda=1$;

- the width $\varepsilon$ is fixed to $\varepsilon=0.1$, i.e. one tenth of the wavelength;

- the angle of incidence is $\theta=\frac{\pi}{3}$.

In Figure 6 we represent the (real part of) the exact total field $u_{t}^{\varepsilon}$. Since the slot is thin, it is difficult to see its effect on the total field in the half space. However one clearly sees the wave that is transmitted inside the slot. 


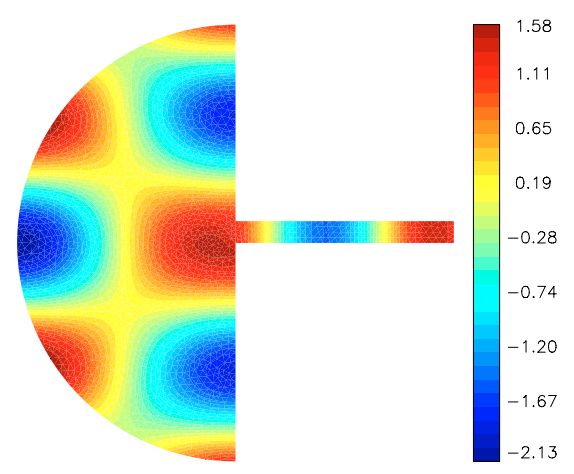

Figure 6. The exact total field.

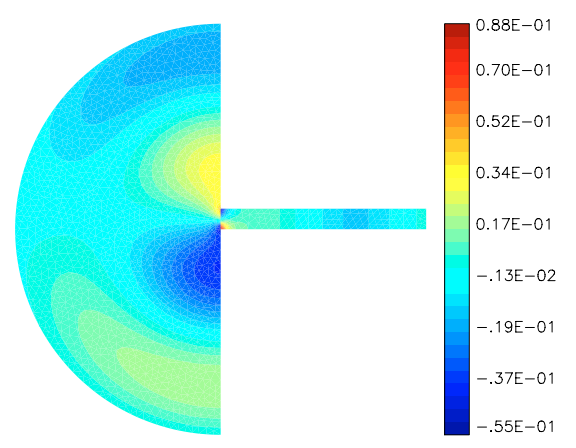

Figure 8. The error due to the model.

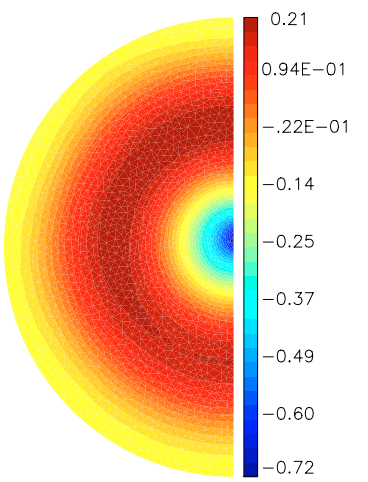

Figure 7. The exact diffracted field.

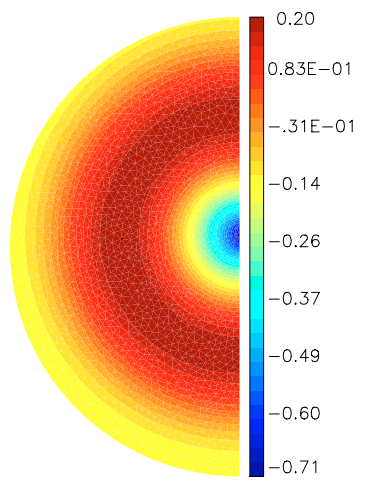

Figure 9. The approximate diffracted field.

In Figure 7, we represent the diffracted field $u^{\varepsilon}=u_{t}^{\varepsilon}-u^{0}$ (which is of course only represented in the halfspace). Note that its amplitude is about 2.5 times smaller than the amplitude on the total field. One can also remark the monopolar structure of this diffracted field: it looks like the response to a point source and is essentially concentrated close to the end of the slot.

In Figure 9, we represent the diffracted field computed with the approximate model, namely $\widetilde{u}^{\varepsilon}$ and the error committed with the approximate model, namely $u^{\varepsilon}-\widetilde{u}^{\varepsilon}$ in Figure 8 . This time the structure of the error is clearly dipolar (i.e. looks like a field created to a point source term which is a first derivative in $y$ of a $\delta$-function). This is due to the fact that the "constant in $y$ " part of the transmitted wave inside the slot is well taken into account by the approximate model. Moreover, this error is once again concentrated close to the end of the slot, even more than the diffracted field itself. Its $L^{\infty}$ norm is about ten times smaller than the one of the diffracted field but this ratio is much smaller if one looks at regions that avoid the end of the slot. This describes what happens for one value of $\varepsilon$. To illustrate our theoretical results we vary $\varepsilon$ and represent the variation of different "errors" as functions of $\varepsilon$. In Figure 10, we compare the diffracted field to the error introduced by the model in the norm of $H^{1}\left(\Omega_{R}\right), R=1$ and we do the same in Figure 11 with the $L^{2}$ norms. The curves are in Log-Log scale and look like straight lines.

Measuring the slope of these lines in Figure 10, we observe that both $u^{\varepsilon}=u_{t}^{\varepsilon}-u^{0}$ and $\widetilde{u}^{\varepsilon}-u^{\varepsilon}$ converge "linearly" to 0 . However $\widetilde{u}^{\varepsilon}-u^{\varepsilon}$ is smaller that $u^{\varepsilon}$ by two orders of magnitude. This illustrate in particular estimates (3.5) and (3.8) for $u_{t}^{\varepsilon}-u^{0}$ and estimate (3.11) for $\widetilde{u}^{\varepsilon}-u^{\varepsilon}$ : graphically, it is almost impossible to detect the presence of the $\sqrt{|\log \varepsilon|}$ term in (3.5). 


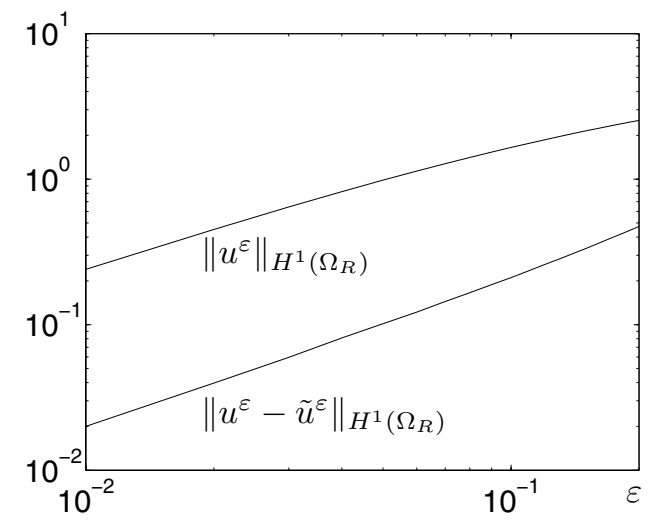

Figure 10. $H^{1}$-global convergence curves.

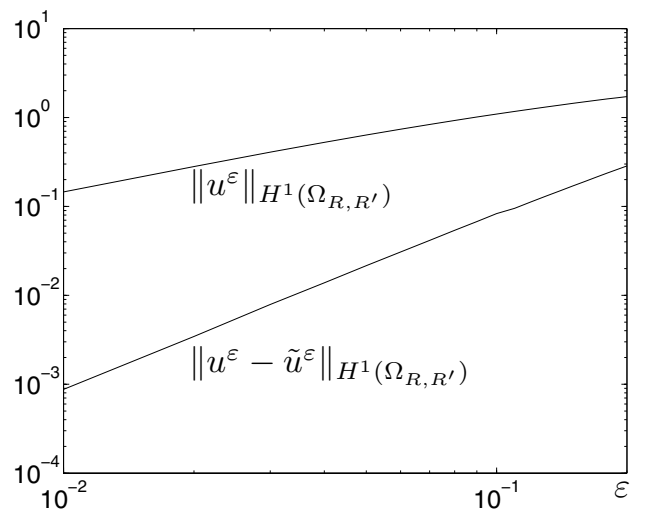

FiguRE 12. $H^{1}$-local convergence curves.

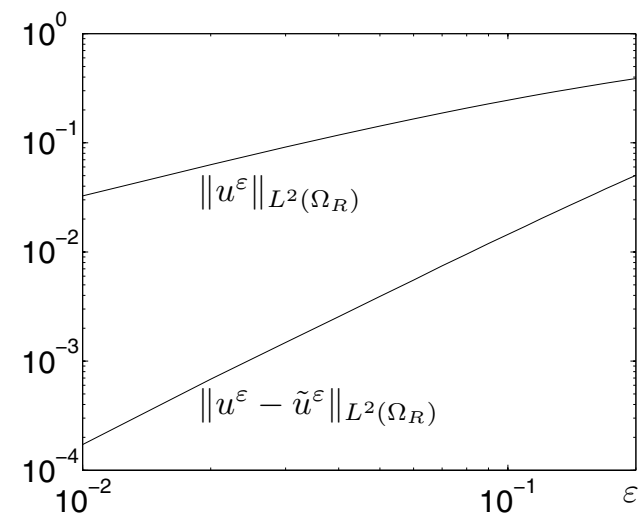

FiguRE 11. $L^{2}$-global convergence curves.

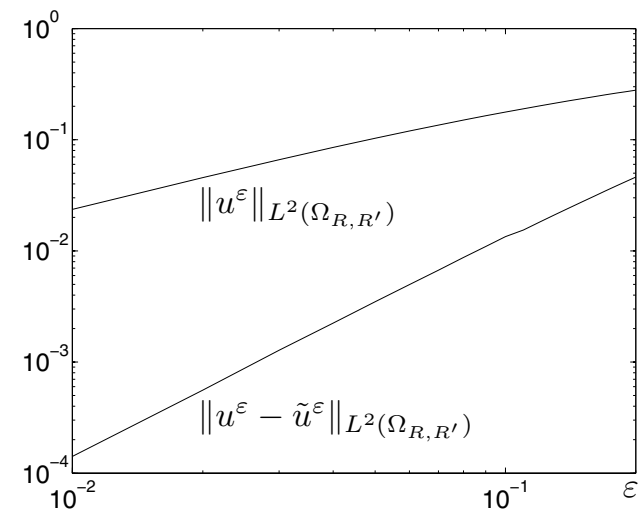

FiguRE 13. $L^{2}$-local convergence curves.

Looking at Figure 11, one clearly sees that, in $L^{2}$ norm, the error $\widetilde{u}^{\varepsilon}-u^{\varepsilon}$ decays much more rapidly to 0 than $u_{t}^{\varepsilon}-u^{0}$. In fact, measuring the slopes, one recovers the $O(\varepsilon)$ estimate for $u_{t}^{\varepsilon}-u^{0}\left(c f\right.$. (3.6)) and the $O\left(\varepsilon^{2}\right)$ estimate for $\widetilde{u}^{\varepsilon}-u^{\varepsilon}(c f .(3.12))$.

In Figures 12 and 13 , we represent the same quantities except that, for computing the norms, we replace the domain $\Omega_{R}$ by $\Omega_{R, R^{\prime}}$ with $R^{\prime}=1 / 2$. Our results illustrate the local estimates. In particular, one sees clearly the gain of one power of $\varepsilon$ by passing from $u^{\varepsilon}=u_{t}^{\varepsilon}-u^{0}$ to $\widetilde{u}^{\varepsilon}-u^{\varepsilon}$ even in the $H^{1}$-norm (contrary to what occurs in the domains $\Omega_{R}$. This illustrates the estimates (3.7) of theorem 3.1 and (3.13) of Theorem 3.3.

\section{Conclusion}

We have proposed in this article an approximate coupling "1D-2D" model for the propagation in media including thin slots. This new model is similar but different to models that have been already used in the literature. The main contribution of this article is a quite complete mathematical analysis of this procedure. Our results and error estimates demonstrate the relevance of the approach but they also point out its limits in terms of accuracy, in particular due to a poor representation of the actual singularities and of the boundary layer of the exact solution. 


\section{REFERENCES}

[1] T. Abboud and F. Starling, Scattering of an electromagnetic wave by a screen, in Boundary value problems and integral equations in nonsmooth domains (Luminy, 1993), Dekker, New York. Lect. Notes Pure Appl. Math. (1995) 167 1-17.

[2] M. Artola and M. Cessenat, Diffraction d'une onde électromagnétique par une couche composite mince accolée à un corps conducteur épais. I. Cas des inclusions fortement conductrices. C. R. Acad. Sci. Paris Sér. I Math. 313 (1991) 231-236.

[3] F. Assous, P. Ciarlet, Jr., and J. Segré, Numerical solution to the time-dependent Maxwell equations in two-dimensional singular domains: the singular complement method. J. Comput. Phys. 161 (2000) 218-249.

[4] J. Beale, Scattering frequencies of reasonators. Comm. Pure Appl. Math. 26 (1973) 549-563.

[5] A. Bendali and K. Lemrabet, The effect of a thin coating on the scattering of a time-harmonic wave for the Helmholtz equation. SIAM J. Appl. Math. 56 (1996) 1664-1693.

[6] A. Bensoussan, J.-L. Lions and G. Papanicolaou, Asymptotic analysis for periodic structures. North-Holland Publishing Co., Amsterdam. Stud. Math. Appl. 5 (1978).

[7] A. Buffa and S.H. Christiansen, The electric field integral equation on Lipschitz screens: definitions and numerical approximation. Numer. Math. 94 (2003) 229-267.

[8] C. Butler and D. Wilton, General analysis of narrow strips and slots. IEEE Trans Ant. and Propag. 28 (1980).

[9] P.G. Ciarlet, Plates and junctions in elastic multi-structures, 14 Recherches en Mathématiques Appliquées [Res. Appl. Math.], Masson, Paris (1990). An asymptotic analysis.

[10] F. Collino and F. Millot, Fils et méthodes d'éléments finis pour les équations de Maxwell. Le modèle de Holland revisité. Tech. Report 3472, INRIA, http://www.inria.fr (Août 1998).

[11] D. Colton and R. Kress, Integral equation methods in scattering theory, John Wiley \& Sons Inc., New York, Pure Appl. Math. (1983). A Wiley-Interscience Publication.

[12] C. Conca and E. Zuazua, Asymptotic analysis of a multidimensional vibrating structure. SIAM J. Math. Anal. 25 (1994) $836-858$.

[13] D. Crighton, A. Dowling, J.F. Williams, M. Heckl and F. Leppington, Modern Methods in Analytical acoustics. Lect. Notes, Springer-Verlag, London (1992). An asymptotic analysis.

[14] R. Dautray and J.-L. Lions, Analyse mathématique et calcul numérique pour les sciences et les techniques. Tome 3. Masson, Paris (1985).

[15] B. Engquist and J.C. Nédélec, Effective boundary conditions for acoustic and electromagnetic scattering in thin layers. Tech. Report 278, École Polytechnique CMAP (France) (1993).

[16] J. Gilbert and R. Holland, Implementation of the thin-slot formalism in the finite-difference code threedii. IEEE Trans. Nuc. Sci. 28 (1981).

[17] D. Givoli, I. Patlashenko and J. Keller, Discrete Dirichlet-to-Neumann maps for unbounded domains. Comput. Methods Appl. Mech. Engrg. 164 (1998) 173-185. Exterior problems of wave propagation (Boulder, CO, 1997; San Francisco, CA, 1997).

[18] P. Grisvard, Elliptic problems in nonsmooth domains 24, Pitman (Advanced Publishing Program), Boston, MA, Monographs Stud. Math. (1985).

[19] P. Grisvard, Singularities in boundary value problems 22, Recherches en Mathématiques Appliquées [Res. Appl. Math.], Masson, Paris (1992).

[20] D. Guiney, B. Noye and E. Tuck, Transmission of water waves through small apertures. J. Fluid Mech. 55 (1972) $149-161$.

[21] I. Harari, I. Patlashenko and D. Givoli, Dirichlet-to-Neumann maps for unbounded wave guides. J. Comput. Phys. 143 (1998) 200-223.

[22] P. Harrington and D. Auckland, Electromagnetic transmission through narrow slots in thick conducting screens. IEEE Trans. Antenna Propagation 28 (1980) 616-622.

[23] R. Holland and L. Simpson, Finite-difference analysis EMP coupling to thin struts and wires. IEEE Trans. Electromagn. Compat. 23 (1981).

[24] P. Joly and S. Tordeux, Modèles asymptotiques pour la propagation des ondes dans les milieux comportant des fentes, Tech. Report RR-5568, INRIA, http://www.inria.fr (May 2005).

[25] J. Keller and D. Givoli, Exact nonreflecting boundary conditions. J. Comput. Phys. 82 (1989) 172-192.

[26] G. Kriegsmann, The flanged waveguide antenna: discrete reciprocity and conservation. Wave Motion 29 (1999) 81-95.

[27] H. Le Dret, Problèmes variationnels dans les multi-domaines 19, Recherches en Mathématiques Appliquées [Res. Appl. Math.], Masson, Paris (1991). Modélisation des jonctions et applications. [Modeling of junctions and applications].

[28] N. Lebedev, Special functions and their applications, Revised English edition. Translated and edited by Richard A. Silverman, Prentice-Hall Inc., Englewood Cliffs, NJ (1965).

[29] J.-L. Lions and E. Magenes, Non-homogeneous boundary value problems and applications. Vol. I, Springer-Verlag, New York (1972). Translated from the French by P. Kenneth, Die Grundlehren der mathematischen Wissenschaften, Band 181.

[30] D. Martin, Documentation de la librairie éléments finis melina. http://perso.univ-rennes1.fr/daniel.martin/melina, 19962003.

[31] P. McIver and A.D. Rawlins, Two-dimensional wave-scattering problems involving parallel-walled ducts. Quart. J. Mech. Appl. Math. 46 (1993) 89-116. 
[32] J.C. Nédélec, Acoustic and electromagnetic equations. Springer-Verlag, New York. Appl. Math. Sci. 144 (2001). Integral representations for harmonic problems.

[33] F. Rogier, Problèmes mathématiques et numériques liés à l'approximation de la géométrie d'un corps diffractant dans les équations de l'électromagnétisme, Ph.D. thesis, Université de Paris 6 (1989).

[34] E. Sánchez-Palencia, Nonhomogeneous media and vibration theory. Lect. Notes Phys. 127 (1980).

[35] T. Senior and J. Volakis, Approximate Boundary Conditions in Electromagnetics. IEE Pres, New York and London (1995).

[36] A. Taflove, Computational electrodynamics. Artech House Inc., Boston, MA (1995). The finite-difference time-domain method.

[37] A. Taflove, K. Umashankar and B. Becker, Calculation and experimental validation of induced currents on coupled wires in an arbitrary shaped cavity. IEEE Trans Antenna Propag. 35 (1987) 1248-1257.

[38] A. Taflove, K. Umashankar, B. Becker, F. Harfoush and K. Yee, Detailed fdtd analysis of electromagnetic fields penetrating narrow slots ans lapped joints in thick conducting screens. IEEE Trans Antenna Propag. 36 (1988) 247-257.

[39] F. Tatout, Propagation d'une onde électromagnétique dans une fente mince. Propagation et réflexion d'ondes en élasticité. Application au contrôle. Ph.D. thesis, École normale supérieure de Cachan (Dec. 1996).

[40] S. Tordeux, Méthodes asymptotiques pour la propagation des ondes dans les milieux comportant des fentes. Ph.D. thesis, Université de Versailles (2005).

[41] E. Tuck, Matching problems involving flow through small holes. Academic Press, New York Adv. Appl. Mechanics 15 (1975) $89-158$.

[42] G. Watson, Bessel functions and Kapteyn series. Proc. London Math. Soc. (April 1916) 150-174. 